\title{
Sur la génération des surfaces et des courbes gauches par les faisceaux de surfaces.
}

\author{
(Par J.-S. et M.-N. V VIVECK, à Jičn - Bohème.)
}

\section{Sur les faisceaux de surfaces.}

1. Une surface $R$ d'ordre $r$ est déterminée par un certain nombre de conditions, qui dépend de l'ordre de la surface $R$. On sait que ce nombre est égal à

$$
\frac{(r+1)(r+2)(r+3)}{1 \cdot 2 \cdot 3}-1
$$

2. Nous allons appeler un système de surfaces qui ne sont pas déterminées par un nombre suffisant de conditions un faisceau de surfaces.

Soit $R$ le nombre de conditions qui déterminent une surface $R$ du rième ordre. Quand la surface $R$ est donnée par $R-n$ conditions, elle forme un faisceau $(R)$.

En prenant $n$ points arbitraires $a, b, c, \ldots$ dans l'espace, on obtiendra un nombre de surfaces $R$ qui correspondent aux $R-n$ conditions données et passent par les points $a, b, c, \ldots$

Appelons le nombre $n$, c'est-à-dire le nombre de points que nous pouvons encore choisir pour compléter les conditions nécessaires à détermination d'une surface $R$, la dimension du faisceau $(R)$ de surfaces $R$.

Le nombre de surfaces $R$ passantrpar les $n$ points $a, b, c, \ldots$ et assujetties aux $R-n$ conditions données nous voulons nommer l'indice du faisceau $(R)$ de la $n^{i e ̀ m e}$ dimension de surfaces $R$.

Alors en disant un faisceau $(R)$ de la $n^{i e m e}$ dimension d'indice $m$ de surfaces $R$ du $r^{\text {ième }}$ ordre, on entend: un système de surfaces $R$ du rième ordre, données par $R-n$ conditions de telle facon, que par $n$ points quelconques $a, b, c, \ldots$ passent $m$ surfaces $R$. 
De là résulte que

$k$ points arbitraires dans l'espace déterminent dans un faisceau de la $n^{i e ̀ m e}$ dimension et d'indice $m$ un faisceau du même indice $m$ mais de la dimension $n-k$.

3. La même chose a lieu quant aux figures planes. Il y a de même dans le plan des faisceaux d'indices divers.

Ainsi quand on dit: un faisceau $(R)$ de la $n^{\text {ieme }}$ dimension et d'indice $m$ de courbes $R$ d'ordre $r$, cela signifie un système de courbes $R$ d'ordre $r$, déterminées par telles conditions que par $n$ points arbitraires du plan passent $m$ courbes $R$.

Il est claire que $k$ points du plan déterminent un faisceau de la dimension $n-k$ et d'indice $m$ dans un faisceau $(R)$ de la $n^{i e ̀ m e}$ dimension et d'indice $m$.

\section{Détermination de l'ordre des surfaces et des courbes.}

4. L'ordre des surfaces. - Dans le Mémoire présent nous nous occuperons avec des surfaces dont l'ordre doit être déterminé, quelque soit le mode de leurs générations.

Pour cet effet il nous suffit une fois pour toutes de démontrer l'ordre de la surface engendrée par la manière suivante.

5. Considérons une surface $S$ comme le lieu de courbes d'intersection de deux autres surfaces appartenant à deux systèmes; ces deux surfaces jouissent de telle propriété que à une d'elles correspond un certain nombre de surfaces du second système rencontrant la première en une courbe de la surface $S$.

Dans ce cas général, il n'est pas nécessaire de connaître ni les ordres des surfaces de deux systèmes ni le nombre de surfaces d'un système correspondant à une surface de l'autre système. Il nous suffit de connaître des données plus simples.

Une droite arbitraire $Q$ perce les surfaces d'un des systèmes en des points $A$ et celles de l'autre système en des points $B$. Supposons que tels points $A$ d'une surface correspondent aux points $B$ de l'autre surface, dont la courbe d'intersection se trouve sur la surface engendrée $S$.

On voit immédiatement que, quand deux points correspondants $A, B$ se réunissent en un seul point, ce point est celui en lequel la droite $Q$ perce la surface $S$.

Il faut que nous déterminions: combien de points $A$ peuvent coïncider 
avec le point correspondant $B$, en sachant, que

$\grave{a}$ un point $A$ pris à volonté sur $Q$ correspondent $b$ points $B$. et réciproquement

que à un point $B$ correspondent a points $A$.

Pour cet effet, faisons passer un plan $P$ par la droite $Q$ et considérons deux points arbitraires $\alpha, \beta$ de ce plan comme les centres de deux faisceaux de droites.

Joignons un point $A$ arec $\alpha$ et le point correspondant $B$ avec $\beta$ par des droites qui se rencontrent en un point. Ce point engendre une courbe $S^{\prime}$ quand les points $A, B$ parcourent la droite $Q$ par la loi indiquée.

La courbe $S^{\prime}$ rencontre $Q$ en des points qui sont évidemment les points de rencontre de la surface $S$ avec la droite $Q$, ou, en d'autres termes, la courbe $S^{\prime}$ et la surface $S$ rencontrent la droite $Q$ en les mêmes points. En déterminant l'ordre de la courbe $S$ ', nous obtenons l'ordre de la surface $S$.

Nous allons déterminer l'ordre de $S^{\prime}$ très-aisément. Un rayon $\alpha^{\prime} \mathrm{du}$ faisceau $(\alpha)$ rencontre $Q$ en un point $A^{\prime}$ auquel correspondent suivant la supposition $b$ points $B$; les jonctions de ces points avec $\beta$ rencontrent $\alpha^{\prime}$ en $b$ points de la courbe $S^{\prime}$.

De là suit que sur un rayon arbitraire $a^{\prime}$ du faisceau (a) se trouvent $b$ points de $S^{\prime}$. Il nous reste encore à examiner si le point a appartient à la courbe $S^{\prime}$.

Dans ce cas un rayon $\beta^{\prime}$ de $(\beta)$ passe par $\alpha$. La droite $\alpha \beta$ rencontre $Q$ en un point $B^{\prime}$ auquel correspondent $a$ points $A$. Par ces points passent les rayons du faisceau $(\alpha)$ correspondant à la droite $\beta^{\prime}$ et rencontrent la en le point $\alpha$. De là suit que le point $\alpha$ appartient à la courbe $S^{\prime}$ et qu'il est multiple d'ordre $a$ ou que la courbe $S^{\prime}$ est d'ordre $a+b$.

Nous avons donc ce théorème:

Une surface $S$ étant le lieu d'intersection de deux figures jouissant de telle proprieté que par un point $A$ pris à volonté sur une droite arbitraire $Q$ passe la première figure et la seconde correspondante rencontre la même droite en $b$ points $B$, et inversement, que par un point $B$ de $Q$ passe la seconde figure pendant que la première correspondante rencontre $Q$ en a points $A$, la surface $S$ est d'ordre $(a+b)$.

6. L'ordre des courbes gauches. - Nous pouvons déterminer l'ordre d'une courbe gauche par une voie semblable à celle que nous avons suivie tout à l'heure.

Supposons que la courbe traitée $K$ soit le lieu des points d'intersection 
d'une courbe $C$ avec une surface $F$, qui changent de positions suivant une certaine loi.

Nous déterminons l'ordre de la courbe $K$, en trouvant le nombre de points qu'elle peut avoir sur un plan arbitraire $Q$.

Ce plan rencontre la courbe $C$ dans une de ses positions en des points $c$ et la surface correspondante $F$ en une courbe $f$. Quand un point $c$ vient d'être placé sur la courbe correspondante $f$, ce point appartient à la courbe examinée $K$.

Afin que nous déterminions le nombre de telles positions du point $c$, qu'il se trouve sur sa courbe correspondante $f$, nous appliquons de nouveau de deux projections.

Projettons les points $c$ d'un point arbitraire $\gamma$ et les courbes $f$ d'un autre point $\varphi$. Une droite projettante $\gamma c$ perce la surface conique correspondante of en des points qui engendrent une courbe auxiliare $L$. Il est clair que chaque point d'intersection de cette courbe avec le plan $Q$ est en même temps le point de rencontre de la courbe $K$ avec $Q$.

De là suit que, en déterminant l'ordre de $L$, nous connaisons de même l'ordre de la courbe $K$, ce qui exige la connaissance des quantités suivantes:

1. le nombre $p$ de courbes $f$ qui correspondent à un point $c$;

$2 .^{\circ}$ le nombre $q$ de points $c$ qui correspondent à une courbe $f$;

3. l'ordre $r$ de la courbe décrite par le point $c$ sur le plan $Q$;

$4 .^{\circ}$ le nombre $t$ de courbes $f$ passant par un point arbitraire du plan $Q$; et enfin

5. l'ordre $f$ de la courbe $f$.

7. Cherchons le nombre de points en lesquels la courbe $L$ rencontre un plan quelconque $P$ passant par le point $\%$ Ce plan rencontre la courbe décrite sur $Q$ par le point $c$ en $r$ points. A chacun de ces points correspondent $p$ courbes $f$ d'ordre $f$. Les surfaces coniques, ayant les courbes $f$ pour lignes directrices et le point $\varphi$ pour sommet, rencontrent les jonctions des points $c$ avec $\gamma$ en des points de la courbe $L$. Le nombre de ces points est donc égal à fpr.

Il nous reste encore à chercher, si le point $\gamma$ appartient à la courbe. Pour cet effet, faisons passer par ce point les surfaces coniques et désignons le point de rencontre de la droite $\gamma \varphi$ avec le plan $Q$ par $d$. Par ce point passent, comme on sait, $t$ courbes $f$, et par conséquent par $\gamma$ passent de même $t$ surfaces coniques $f \varphi$.

A chacune de ces surfaces correspondent $q$ points $c$ ainsi que des rayons $\gamma c$, 
dont chacun détermine sur la surface conique correspondante le point $\gamma$ comme le point de la courbe $L$.

Il s'ensuit que $\gamma$ est un point multiple d'ordre $q t$ de la courbe $L$ et que cette ligne est d'ordre

$$
\operatorname{frp}+q t
$$

parce que le plan $Q$ la coupe en autant de points.

La courbe $K$ est du même ordre. De là résulte ce théorème:

Quand les points d'une courbe $K$ proviennent de l'intersection d'une courbe $C$ avec une surface $F$ qui forment deux systèmes correspondants de telle manière que, en coupant $C$ en des points $c$ et $F$ en une courbe $f$ par un plan arbitraire $Q$,

la courbe $f$ soit d'ordre $f$;

$t$ courbes $f$ passent par un point quelconque du plan $Q$;

le point $c$ décrit une courbe d'ordre $r$ sur $Q$;

$\grave{a}$ une courbe $f$ correspondent $q$ points $c$; et enfin

$\grave{a}$ un point $c$ correspondent $p$ courbes $f$, alors la courbe $K$ est d'ordre

$$
f p r+q t \text {. }
$$

8. L'ordre d'une courbe plane. - Supposons que nous obtenons les points d'une courbe plane comme les points d'intersection des courbes correspondantes de deux faisceaux distincts.

Pour déterminer l'ordre de cette courbe, nous pouvons employer le même procédé que nous avons appliqué à une surface.

Les courbes d'un des faisceaux donnés rencontrent une droite arbitraire du plan en des points $A$ et celles de l'autre faisceau en des points $B$.

Prenons un point $A$ sur $Q$. Les courbes correspondantes de l'autre faisceau rencontrent la en $b$ points $B$ et réciproquement à un point $B$ correspondent $a$ points $A$.

Ainsi: d'ordre

Le lieu des points d'intersection de deux faisceaux donnés est une courbe

$$
a+b
$$

Nous pouvons de là déduire une formule qui peut être employée directement.

9. Considérons deux faisceaux $\left(F_{1}\right),\left(F_{2}\right)$ de la première dimension et d'indices $m_{1}, m_{2}$ de courbes $F_{1}, F_{2}$ d'ordre $f_{1}, f_{2}$, dont les points de rencontre remplissent une courbe. 
Quand à une courbe $F_{1}$ correspondent $a$ courbes $F_{2}$ et à une courbe $F_{2}$ correspondent $b$ courbes $F_{1}$, la courbe dérivée doit être d'ordre

$$
a f_{1} m_{1}+b f_{2} m_{2} \text {. }
$$

En désignant le nombre de points obtenus directement sur une courbe quelconque du faisceau $\left(F_{1}\right)$ par $F_{1}$, nous pouvons écrire

$$
\frac{a f_{1} f_{2}}{f_{1}} \text { au lieu de } a f_{2}
$$

et parce que $a f_{1} f_{2}=F_{1}$ nous avons

$$
a f_{2}=\frac{F_{1}}{f_{1}} .
$$

En faisant usage de cette notion aussi pour le second faisceau $\left(F_{2}\right)$, nous obtenons que l'ordre de la courbe dérivée est

$$
m_{1} \frac{F_{1}}{f_{1}}+m_{2} \frac{F_{2}}{f_{2}} .
$$

De là suit ce théorème:

Une courbe $C$ est le lieu des points de rencontre des courbes d'ordres $f_{1}, f_{2}$ de deux faisceaux $\left(F_{1}\right),\left(F_{2}\right)$ de la première dimension et d'indices $m_{1}, m_{2} . F_{1}, F_{2}$ soient les nombres de points obtenus directement sur deux courbes arbitraires $F_{1}, F_{2}$ des faisceaux $\left(F_{1}\right),\left(F_{2}\right)$. L'ordre de la courbe $C$ est égal à la somme de deux termes, dont chacun est construit ainsi: on divise le nombre de points obtenus directement sur une courbe d'un des faisceaux donnés par l'ordre de cette courbe et on multiplie ce quotient par l'indice du faisceau auquel appartient cette courbe.

10. L'importance de ce théorème se manifeste principalement dans le cas, quand la courbe $C$ provient de l'intersection des tangentes correspondantes de deux courbes $A, B$ de classe $\alpha, \beta . a, b$ étant les nombres de points de la courbe $C$, que nous obtenons directement sur une tangente arbitraire respectivement de la courbe $A, B$, l'ordre de la courbe $C$ est

$$
\alpha a+\beta b .
$$

\section{Sur la surface engendrée par les points d'intersection de $n$ surfaces.}

11. Soit donné un faisceau $(R)$ de surfaces $R$ d'ordre $r$; supposons que ce faisceau est de la dimension $n-1$ et d'indice $m_{r}$. Outre cela soient données les courbes

$$
\left(p_{1}\right), \quad\left(p_{2}\right), \ldots \quad\left(p_{n}\right)
$$


à chacune de ces courbes correspond un faisceau de surfaces de la première dimension; nous avons respectivement les faisceaux

$$
\left(F_{1}\right), \quad\left(F_{2}\right), \ldots\left(F_{n}\right) .
$$

Une surface $R$ du faisceau $(R)$ rencontre les courbes $\left(p_{1}\right),\left(p_{2}\right), \ldots$ en des points. Aux points ainsi obtenus sur $\left(p_{1}\right)$ faisons correspondre les points sur

et réciproquement.

$$
\left(p_{2}\right), \quad\left(p_{3}\right), \ldots\left(p_{n}\right),
$$

Ces points déterminent les surfaces dans les faisceaux correspondants. Supposons que les surfaces passant par les points correspondants se correspondent de même. Prenons une à une des surfaces correspondantes; ces $n$ surfaces $T$ se rencontrent en des points.

Quand la surface $R$ forme le dit faisceau $(R)$, il peut arriver que entre les points, dont nous venons de parler, se trouvent tels points que toutes les $n$ surfaces correspondantes passent par ces points.

Quel est le lieu de ces points?

Pour déterminer ce lieu, considérons le cas quand $n=3$. Il est clair que, quand nous prenons trois telles surfaces correspondantes $F$, c'est-à-dire une surface $F_{1}$, une surface $F_{2}$ et une surface $F_{3}$, ces surfaces correspondantes se rencontrent en des points, par chacun d'eux passent toutes ces trois surfaces $F_{1}, F_{2}, F_{3}$.

Afin que nous trouvions le lieu de ces points, il faut montrer que sur une droite arbitraire se trouve un nombre fini de ces points ou qu'ils remplisse une surface.

12. Nous voulons d'abord démontrer que, $n$ étant égal à 2 , la courbe d'intersection de deux surfaces correspondantes $F_{1}, F_{z}$ engendre une surface.

Une droite arbitraire $Q$ rencontre les surfaces $F_{1}, F_{2}$ en des points que nous désignons respectivement par $A, B$. Les points $A$ correspondent aux points $B$.

Considérons un point pris à volonté sur la droite $Q$ comme le point $A$. Par ce point passe un certain nombre de surfaces $F_{\mathfrak{i}}$, dont chacune détermine sur $\left(p_{1}\right)$ de même un nombre fixe de points; par chacun d'eux passe un certain nombre de surfaces $R$. Chacune de ces surfaces détermine sur $\left(p_{2}\right)$ des points qui déterminent des surfaces $F_{2}$ rencontrant la droite $Q$ en des points $B$ correspondant au point $A$.

A un point $A$ correspond, par conséquent, un nombre fixe de points $B$ et, parce que cela a lieu aussi réciproquement, on voit, qu'il y a des points en 
lesquels coïncident les points $A$ arec leurs points correspondants $B$. Ce sont évidemment les points en lesquels la droite $Q$ perce la surface engendrée par les courbes d'intersection des surfaces $F_{1}, F_{2}$.

13. La même voie nous conduira au but demandé dans le cas de l'article 11. Un point quelconque $p_{3}$ sur $\left(p_{3}\right)$ détermine dans le faisceau $(R)$ un faisceau de la première dimension.

En considérant la surface $F_{3}$ passant par le point $p_{3}$, la courbe d'intersection des surfaces correspondantes $F_{1}, F_{2}$ engendre, d'après le précédent, une surface qui rencontre la surface $F_{3}$ en une courbe d'une figure nouvelle. Nous montrerons que cette dernière figure rencontre la droite $Q$ en des points, ou, en d'autres termes, qu'elle est une surface.

Supposons que la surface $F_{3}$ rencontre $Q$ en des points $A$, et que la surface engendrée par les autres surfaces correspondantes coupe cette droite en des points $B$.

Par un point $A$ pris à volonté sur $Q$ passent des surfaces $F_{3}$ qui déterminent sur $\left(p_{3}\right)$ les points $p_{3}$. Ces points déterminent des faisceaux de la première dimension; à chacun d'eux correspond une surface auxiliaire rencontrant $Q$ en des points $B$.

A un point $A$ correspond donc un nombre fixe de points $B$, et réciproquement. Car un point arbitraire $B$ sur $Q$ détermine les surfaces $F_{1}, F_{2}$ qui rencontrent les courbes $\left(p_{1}\right),\left(p_{2}\right)$ respectivement en des points $p_{1}, p_{2}$.

Chacun des points $p_{1}$ détermine arec un des points $p_{2}$ une surface $R$ rencontrant $\left(p_{3}\right)$ en des points qui déterminent les surfaces $F_{3}$. Ces surfaces coupent la droite $Q$ en des points $A$.

C. Q. F. D.

Par conséquent nous obtenons sur $Q$ de nouveau des points en lesquels coïncide un point $A$ avec son point correspondant $B$. Il est clair que ce sont les points en lesquels la droite $Q$ perce la figure engendrée par les points d'intersection des surfaces correspondantes $F_{1}, F_{2}, F_{3}$.

14. En connaissant ces résultats pour $n=2$ et $n=3$, nous pourons les étendre à un nombre arbitraire, c'est-à-dire nous pouvons démontrer que ces résultats sont valables pour $n$ quand cela a lieu pour $n-1$.

Considérons alors un faisceau $R$ de la dimension $n-2$, puis $n-1$ courbes $p_{1}, p_{2}, \ldots p_{n-1}$ et enfin autant de faisceaux $\left(F_{1}\right),\left(F_{2}\right), \ldots\left(F_{n-1}\right)$ correspondants de la première dimension. Supposons qu'il y a toujours un certain nombre de points par lesquels passent toutes $n-1$ surfaces correspondantes $F_{1}, F_{2}, F_{n-1}$, et que tous ces points remplissent une surface $S_{n-1}$.

Considérons maintenant un faisceau $(R)$ de la dimension $n-1$, et ajoutons encore une courbe $\left(p_{n}\right)$ et son faisceau correspondant $\left(F_{n}\right)$. 
Une droite arbitraire $Q$ rencontre les surfaces $F_{n}$ en des points $A$. En prenant le point $p_{n}^{\prime}$ sur $\left(p_{n}\right)$, par lequel passe une telle surface $F_{n}$, pour point du faisceau $(R)$, nous obtenons un nouveau faisceau de la $(n-2)^{\text {ième dimension, }}$ duquel nous pouvons déduire une nouvelle surface $S_{n-1}$ qui rencontre $Q$ en des points $B$.

Nous voyons que, en prenant un point $A$ sur $Q$, par ce point passe un nombre fixé de surfaces $F_{n}$ qui rencontrent la courbe $\left(p_{n}\right)$ en des points déterminant les faisceaux $(R)$ de la dimension $n-2$. Ces faisceaux déterminent des surfaces $S_{n-1}$ qui rencontrent la droite $Q$ en des points $B$.

Il suit de Jà que à un point $A$ correspond un nombre fini de points $B$.

La même chose a lieu quand nous commençons par un point $B$, qui détermine des surfaces dans les faisceaux $\left(F_{1}\right),\left(F_{2}\right), \ldots\left(F_{n-1}\right)$ rencontrant leurs courbes correspondantes $\left(p_{1}\right),\left(p_{2}\right), \ldots\left(p_{n-1}\right)$ en des points. En prenant un à un de ces points sur chaque courbe, il détermine une ou plusieurs surfaces $R$ qui fournissent les points $p_{n}$ par lesquels passent les surfaces $F_{n}$ déterminant les points correspondants $A$.

Nous voyons donc que à un point $B$ correspond un nombre fini de points $A$. Il y a ainsi des points $A$ qui coïncident avec leurs points correspondants $B$. Ces points sont évidemment ceux, en lesquels la droite $Q$ rencontre le lieu des points provenant de l'intersection des surfaces correspondantes $F$; ce que nous pouvons définir ainsi:

Il y a en vérité un nombre infini de points dans l'espace par lesquels passent simultanément les surfaces correspondantes de tous faisceaux; ces points remplissent une surface.

\section{L'ordre de la surface $S_{2}$.}

15. La même voie, que nous avons suivie en démontrant que le lieu des points de rencontre de toutes les surfaces correspondantes des faisceaux $(F)$ est une surface $S_{n}$, nous conduit à la détermination de l'ordre $s_{n}$ de cette surface.

Nous allons dériver une formule, dont nous pouvons démontrer qu'elle est valable pour $n$, quand elle est correcte pour $n-1$. Commençons par $n=2$.

16. Soient données:

un faisceau $(R)$ de la première dimension et d'indice $m_{2}$ de surfaces $R$ du $r^{\text {ième }}$ ordre;

Annali di Matematica, tomo XIV. 
les courbes $\left(p_{1}\right),\left(p_{2}\right)$ d'ordres $p_{1}, p_{2}$; et enfin

les faisceaux $\left(F_{1}\right),\left(F_{2}^{\prime}\right)$ de la première dimension, d'indices $m_{1}, m_{2}$ de surfaces d'ordres $f_{1}, f_{2}$.

Une surface $R$ rencontre les courbes $\left(p_{1}\right),\left(p_{2}\right)$ en $r p_{1}$ points $p_{1}$ et en $r p_{2}$ points $p_{2}$. Chaque point $p_{1}$ détermine $m_{1}$ surfaces $F_{1}$ et chaque $p_{2}$ détermine $m_{2}$ surfaces $F_{2}$.

Les surfaces $F_{1}$, ainsi obtenues, rencontrent les surfaces $F_{2}$, qui leurs eorrespondent suivant la loi indiquée, en des courbes qui remplissent une surface $S_{2}$. L'ordre de cette surface peut être déterminé de la manière que nous avons exposée dans l'article 4.

Supposons que les surfaces $F_{1}$ rencontrent $Q$ en des points $A$ et leurs surfaces correspondantes la coupent en des points $B$. Un point arbitraire $A$ sur $Q$ détermine $m_{1}$ surfaces $F_{1}$ du faisceau $\left(F_{1}\right)$ rencontrant la courbe $\left(p_{1}\right)$ en $f_{1} m_{1} p_{1}$ points $p_{1}$. Chacun d'eux détermine $m_{2}$ surfaces $R$ coupant la courbe $\left(p_{2}\right)$ en

$$
f_{1} m_{1} m_{r} p_{1} p_{2} r
$$

points $p_{2}$, dont chacun détermine $m_{2}$ surfaces $F_{2}$ qui coupent, par conséquent, la droite $Q$ en

$$
f_{1} f_{2} m_{1} m_{2} m_{r} p_{1} p_{2} r
$$

points $B$ correspondant au point $A$. Nous obtenons par le même raisonnement que à un point $B$ correspondent

points $A$.

$$
f_{1} f_{2} m_{1} m_{2} m_{r} p_{1} p_{2} r
$$

Nous trouvons ainsi

$$
2 f_{1} f_{2} m_{1} m_{2} m_{r} p_{1} p_{2} r
$$

points $A$ sur $Q$, qui coïncident avec leurs points correspondants $B$. Ces points sont évidemment ceux, en lesquels la droite $Q$ perce la surface $S_{2}$. Cette surface est donc d'ordre

$$
s_{2}=2 f_{1} f_{2} m_{1} m_{2} m_{r} p_{1} p_{2} r .
$$

Pour abréger, nous écrirons dans ce qui va suivre les produits partiels ainsi:

$$
\begin{aligned}
& F_{n}=f_{1} f_{2} \ldots f_{n} \\
& M_{n}=m_{1} m_{2} \ldots m_{n} \\
& P_{n}=p_{1} p_{2} \ldots p_{n} .
\end{aligned}
$$

En faisant usage de cette notation, nous pouvons donc écrire la formule précédente comme il suit:

$$
2 r m_{r} F_{2} M_{2} P_{2} \text {. }
$$




\section{L'ordre de la surface $S_{n}$.}

17. Quand nous avons résolu le problème pour $n=2$, nous pouvons le faire aussi quand $n$ est d'une grandeur arbitraire.

Supposons que soient données: un faisceau $(R)$ de la dimension $n-1$ et d'indice $m_{r}$ de surfaces $R$ d'ordre $r$; les faisceaux $\left(F_{1}\right),\left(F_{2}\right), \ldots\left(F_{n}\right)$ de la première dimension et d'indices $m_{1}, m_{2}, \ldots m_{n}$ de surfaces $F_{1}, F_{2}, \ldots F_{n}$ respectivement d'ordre $f_{1}, f_{2}, \ldots f_{n}$, et enfin les courbes $\left(p_{1}\right),\left(p_{2}\right), \ldots\left(p_{n}\right)$ d'ordres $p_{1}, p_{2}, \ldots p_{n}$ à l'aide desquelles nous établissons la correspondance des surfaces $F_{1}, F_{2}, \ldots F_{n}$.

18. La construction de la surface $S_{22}$ est la suivante. Une surface $R$ rencontre les courbes $\left(p_{1}\right),\left(p_{2}\right), \ldots\left(p_{n}\right)$ en points correspondants de telle manière que aux points, ainsi obtenus sur $\left(p_{1}\right)$, correspondent tous les points déterminés par la même surface $R$ sur les autres courbes.

Sur chacune des courbes on prend un à un point correspondant qui détermine dans le faisceau $(F)$ correspondant des surfaces qui sont appelées les correspondantes, quand elles passent par les points correspondants.

Quand $n$ de ces surfaces correspondantes, prises une à une dans chacun des faisceaux, passent par un point $s$, ce point appartient à la surface $S_{n}$.

19. Considérons de nouveau une droite arbitraire $Q$. En prenant sur $\left(p_{n}\right)$ un point $p_{n}$, par ce point passent $m_{n}$ surfaces $\left(F_{n}\right)$ qui rencontre $Q$ en des points $A$.

Le point $p_{n}$ détermine dans le faisceau $(R)$ un faisceau de la $(n-2)^{\text {ièms }}$ dimension, duquel on peut déduire à l'aide des courbes $\left(p_{1}\right),\left(p_{2}\right), \ldots\left(p_{n-1}\right)$ et des faisceaux $\left(F_{1}\right),\left(F_{2}\right), \ldots\left(F_{n-1}\right)$ une nouvelle surface $S_{n-1}$ qui rencontre la droite $Q$ en des points $B$. Nous allons déterminer le nombre de point $B$ correspondant à un point arbitraire $A$, et viceversa.

Considérons un point $A$ sur $Q$. Par ce point passent $m_{n}$ surfaces $F_{n} d u$ faisceau $\left(F_{n}\right)$, qui coupent la courbe $\left(p_{n}\right)$, en général, en $f_{n} m_{n} p_{n}$ points, dont chacun détermine dans le faisceau $(R)$ un faisceau de la $(n-2)^{\text {ièmo }}$ dimension déterminant la surface $S_{n-1}$ qui rencontre $Q$ en $s_{n-1}$ points $B$ correspondant au point $A$.

$A$ un point $A$ correspondent donc

points $B$.

$$
f_{n} m_{n} p_{n} s_{n-1}
$$

Suivons la voie inverse et prenons sur $Q$ un point $B$. Ce point détermine $m_{1}$ surfaces $F_{1}$ dans le faisceau $\left(F_{1}\right)$ rencontrant la courbe $\left(p_{n}\right)$ en général en $f_{1} m_{1} p_{1}$ points $p_{1}$. 
Le point $B$ détermine de même $m_{2}$ surfaces $F_{2}$ dans le faisceau $\left(F_{2}\right)$, qui coupent la courbe $\left(p_{2}\right)$ en $f_{2} m_{2} p_{2}$ points, etc. Dans le faisceau $\left(F_{n-1}\right)$ nous obtenons $m_{n-1}$ surfaces $F_{n-1}$ qui rencontrent $\left(p_{n-1}\right)$ en $f_{n-1} m_{n-1} p_{n-1}$ points $p_{n-1}$. Prenons de tous ces points correspondants un à un sur chaque courbe $(p)$. Ces $n-1$ points déterminent $m_{r}$ surfaces $R$ dans le faisceau $(R)$. Il est clair que nous obtenons de tels groupes à $n-1$ points en général

ou abrégé

$$
f_{1} f_{2} \ldots f_{n-1} m_{1} m_{2} \ldots m_{n-1} p_{1} p_{2} \ldots p_{n-1}
$$

$$
F_{n-1} M_{n-1} P_{n-1} \text {. }
$$

Chacun de ces groupes détermine $m$ surfaces $R$ dont chacune rencontre $\left(p_{n}\right)$ en $r p_{n}$ points. Par chacun d'eux passent $m_{n}$ surfaces $F_{n}$ qui coupent la droite $Q$ en des points $A$ correspondant au point $B$.

$\grave{A}$ un point $B$ correspondent done

points $A$.

$$
m_{r} r F_{n} M_{n} P_{n}
$$

Nous voyons ainsi que sur la droite $Q$ se trouvent

$$
f_{n} m_{n} p_{n} s_{n-1}+m_{r} r F_{n} M_{n} P_{n}
$$

points en lesquels coïncident les points $A$ avec leurs points correspondants $B$, qui sont évidemment les points d'intersection de la droite $Q$ avec la surface $S_{n}$. Cette surface est donc d'ordre

$$
s_{n}=m_{r} r F_{n} M_{n} P_{n}+f_{n} m_{n} p_{n} s_{n-1} .
$$

Parce que pour $n=2$

et pour $n=3$

$$
\begin{aligned}
& s_{2}=2 r m_{r} F_{2} M_{2} P_{2} \\
& s_{3}=3 r m_{r} F_{3} M_{3} P_{3}
\end{aligned}
$$

d'où l'on peut conclure que la formule générale ait la forme

$$
s_{n}=n r m_{r} F_{n} M_{n} P_{n},
$$

car si elle est valable pour $s_{n-1}$, on obtiendra pour $n$

$$
s_{n}=m_{r} \cdot F_{n} M_{n} P_{n}+f_{n} m_{n} p_{n} r F_{n-1} M_{n-1} P_{n-1}
$$

ce qui prouve que la formule précédente est exacte, puisqu'elle est correcte pour $n=3$; ainsi

$$
s_{n}=n m_{r} r F_{n} M_{n} P_{n}
$$




\section{Cas particuliers.}

20. Quand $n=3$ nous avons ce théorème:

Un faisceau de la seconde dimension de surfaces détermine des points sur trois courbes, par lesquels passent les surfaces de leurs faisceaux correspondants; ces surfaces se rencontrent en des points d'une surface nouvelle qui est d'ordre

$$
s_{3}=3 m_{r} r F_{n} M_{n} P_{n} .
$$

21. Supposons que les surfaces de tous les faisceaux sont du second ordre, que les faisceaux sont d'indice 1 et que les lignes $(p)$ deviennent des droites; alors

$$
s_{3}=3 \cdot 2 \cdot 2^{3}=48 \text {. }
$$

22. Considérons une surface appartenant au faisceau $\left(F_{1}\right)$ et une autre surface du faisceau $\left(F_{2}\right)$; ces surfaces se rencontrent en une courbe du quatrième ordre dont les points d'intersection avec la surface $S_{3}$ nous allons déterminer.

Les surfaces $F_{1}, F_{3}$ rencontrent respectivement les droites $\left(p_{1}\right),\left(p_{2}\right)$ en des points qui, étant quatre, déterminent quatre groupes de deux à deux points, et par conséquent ils déterminent autant de surfaces $R$, dont chacune rencontre la droite $\left(p_{3}\right)$ en deux points déterminant une à une surface $F_{3}$.

Il suit de là que nous obtenons ainsi sur la dite courbe d'intersection du quatrième ordre directement

points.

$$
8 \cdot 8=64
$$

Seulement il faut que sur cette courbe soient $4 \cdot 48=192$ tels points. Outre cela, on voit que ce sont tous les points qui résultent de surfaces générales du second ordre (tous les faisceaux sont donc du premier indice).

De là suit que les points de rencontre de la dite courbe du quatrième ordre avec les courbes fondamentales des faisceaux $\left(F_{1}\right),\left(F_{2}\right)$ appartiennent à la courbe considérée. Il y en a seize. Ils sont, par conséquent, les points multiples d'ordre 8. Nous voyons done que les courbes fondamentales des faiseeaux $\left(F_{1}\right),\left(F_{2}\right)$ sont les lignes multiples d'ordre 8 sur la surface $S_{3}$, ce que nous trouverons justifié plus loin.

23. Toutes les conditions restent les mêmes comme précédemment, seulement $n=2$; la surface $S_{2}$ est d'ordre

$$
s_{2}=2 \cdot 2 \cdot 2^{2}=16 .
$$


Une surface $F_{1}$ du faisceau $\left(F_{1}\right)$ rencontre la droite $\left(p_{1}\right)$ en deux points qui déterminent deux surfaces $R$, dont chacune coupe la droite $\left(p_{2}\right)$ en deux points. Chacun d'eux détermine une surface $F_{2}$. Sur la surface $F_{1}$ se trouvent donc quatre courbes du quatrième ordre de la surface $S_{2}$. Il nous manque encore une partie de la courbe d'intersection des surfaces $F_{1}, S_{2}$. C'est la courbe fondamentale du faisceau $\left(F_{1}\right)$, qui est une ligne multiple d'ordre 4 sur la surface $S_{2}$; de même la courbe fondamentale du faisceau $\left(F_{2}\right)$ est une ligne multiple d'ordre 4 sur $S_{2}$.

24. Quand le faisceau $(R)$ consiste en des plans, nous obtenons dans le premier cas une surface d'ordre 16 , ayant les courbes fondamentales des faicceaux $\left(F_{1}\right),\left(F_{2}\right)$ pour lignes multiples d'ordre 4 et dans le second cas une surface $S_{2}$ du huitième ordre, qui possède les courbes fondamentales des faisceaux $\left(F_{1}\right),\left(F_{2}\right)$ comme les lignes multiples d'ordre 2 .

25. Dans le cas que les surfaces $R$ et $F$ sont des plans et $n=2$, nous retrouvons la génération bien connue des surfaces gauches au moyen de trois surfaces développables des classes $m_{1}, m_{2}, m_{3}$. L'ordre de la surface engendrée est d'après la formule générale

$$
s_{2}=2 m_{1} m_{2} m_{3},
$$

ce qui est d'accord arec le résultat connu.

26. Si tous les faisceaux étaient les faisceaux de plans ou les surfaces développables, $n$ étant égal à 3 , nous obtenons une surface engendrée par les points, qui est d'ordre

$$
s_{3}=3 m_{1} m_{2} m_{3} m_{r}
$$

et quand les faisceaux de plans sont les faisceaux ordinaires, la surface obtenue est une surface générale du troisième ordre.

Nous somme parvenu à ces deux résultats par une autre voie, c'est-à-dire par un mode analogue au procédé de Mac-Laurin.

27. Considérons le cas intéressant quand $n=4$ et $(R)$ est un faisceau de plans de la troisième dimension ou, en d'autres termes, quand $(R)$ est le système de plans qui remplissent tout l'espace indéfini.

Quand

et

$$
f_{1}=f_{2}=f_{3}=f_{1}=2, \quad m_{1}=m_{2}=m_{3}=m_{4}=1
$$

la surface $S_{4}$ est d'ordre

$$
p_{1}=p_{2}=p_{3}=p_{1}=1,
$$

$$
s_{4}=4 \cdot 2^{4}=64
$$


Nous pouvons de même démontrer, comme dans le précédent, que les courbes fondamentales des faisceaux $(F)$ sont les lignes multiples d'ordre 8 de la surface $S_{1}$.

28. Considérons enfin le cas quand $n=4$ et toutes les autres quantités sont égaux à 1 ; la surface engendrée est du quatrième ordre.

Les points de cette surface jouissent de telle propriété que par chacun d'eux passe un à un des plans de quatre faisceaux de plans, qui rencontrent leurs quatre droites correspondantes en des points qui sont situés sur un même plan.

En prenant deux plans $F_{1}, F_{2}$ des faisceaux $\left(F_{1}\right),\left(F_{2}\right)$, ces plans se rencontrent en une droite et coupent les droites $\left(p_{1}\right),\left(p_{2}\right)$ en les points $p_{1}, p_{2}$. Ces points déterminent un faisceau de plans $(R)$ de la première dimension, duquel on peut déduire une surface réglée du second ordre, qui rencontre la droite $F_{1}, F_{2}$ en des points de la surface $S_{4}$.

Il est clair que les axes des faisceaux $\left(F_{1}\right),\left(F_{2}\right)$ et par conséquent de même ceux des faisceaux $\left(F_{3}\right),\left(F_{4}\right)$ sont les génératrices de la surface $S_{4}$.

\section{Sur la courbe qui est le lieu des points d'intersection des surfaces de trois faisceaux.}

29. La courbe gauche peut être engendrée par la même manière comme la surface. Considérons d'abord seulement trois faisceaux de surfaces $F$, ce qui est le cas le plus simple. Soient données: d'ordre $r$

un faisceau $(R)$ de la première dimension, d'indice $m_{r}$ de surfaces

trois courbes $\left(p_{1}\right),\left(p_{2}\right),\left(p_{3}\right)$ respectivement d'ordre $p_{1}, p_{2}, p_{3}$; et enfin trois faisceaux $\left(F_{1}\right),\left(F_{2}\right),\left(F_{3}\right)$ de surfaces, qui sont de la première dimension et d'indices $m_{1}, m_{2}, m_{3}$, les surfaces $F_{1}, F_{2}, F_{3}$ étant respectivement d'ordre $f_{1}, f_{2}, f_{3}$.

Une surface arbitraire $R$ du faisceau $(R)$ rencontre de même les courbes $\left(p_{1}\right),\left(p_{2}\right),\left(p_{3}\right)$ en des points qui se correspondent de telle manière que les points ainsi obtenus sur une de ces courbes correspondent aux points sur les autres courbes.

Chacun de ces points d'intersection de la surface $R$ avec les courbes $(p)$ détermine un nombre de surfaces dans le faisceau correspondant $(F)$; les surfaces passant par les points correspondants soient appelées de même les surfaces correspondantes. 
En prenant dans chacun des faisceaux une à une surface correspondante, ces trois surfaces $F_{1}, F_{2}, F_{3}$. se rencontrent on des points qui engendrent une courbe, quand la surface $R$ engendre le faisceau $(R)$.

30. Déterminons les points d'intersection de cette courbe avec un plan arbitraire $Q$ par le procédé que nous avons indiqué en 4 .

Une surface quelconque $F_{1}$ rencontre $\left(p_{t}\right)$ en des points, dont chacun détermine des surfaces $(R)$. Chacune d'elles coupe la courbe $\left(p_{2}\right)$ en des points $p_{2}$ et la courbe $\left(p_{3}\right)$ en des points $p_{3}$. Les surfaces du faisceau $\left(F_{2}\right)$ passant par les points $p_{2}$ et celles du faisceau $\left(F_{3}\right)$ passant par $p_{3}$ sont toutes les surfaces qui correspondent à la surface $F_{1}$ prise à volonté dans le faisceau $\left(F_{1}\right)$.

Ces surfaces $F_{2}, F_{3}$ se rencontrent une à une en une courbe $\left(F_{2} F_{3}\right)$ qui perce la dite surface $F_{1}$ en des points de la courbe $C_{3}$. Nous nous servirons de ce mode de génération de la courbe $C_{3}$ à la détermination de son ordre.

Supposons que la surface $F_{1}$ rencontre le plan $Q$ en une courbe $f$ et la courbe $\left(F_{2} F_{3}\right)$ le coupe en un point $c$. Si nous considérons un point du plan $Q$ comme le point $c$, nous savons qu'il résulte de l'intersection de ce plan avec une certaine courbe $\left(F_{2} F_{3}\right)$, et, par conséquent, qu' il correspond à une certaine surface $R$ qui rencontre $\left(p_{1}\right)$ en $r p_{1}$ points dont chacun détermine $m_{1}$ surfaces $F_{1}$ et chacune d'elles détermine une courbe $f$. Donc

$\grave{a}$ un point $c$ correspondent $m_{1} p_{1} r$ courbes $f$.

En prenant une surface $F_{1}$ et par suite aussi une courbe $f$, cette surface rencontre $\left(p_{1}\right)$ en $f_{1} p_{1}$ points qui déterminent $m_{2} f_{1} p_{1}$ surfaces $R$, dont chacune rencontre $\left(p_{2}\right)$ en $r p_{2}$ points $p_{2}$ et $\left(p_{3}\right)$ en $r p_{3}$ points $p_{3}$. Chaque point $p_{2}$ détermine $m_{2}$ surfaces $F_{2}$ et chaque points $p_{3}$ détermine $m_{3}$ surfaces $F_{3}$.

Ces surfaces $F_{2}, F_{3}$ se rencontrent en

$$
m_{r} m_{2} m_{3} r^{2} p_{1} p_{2} f_{1}
$$

courbes $\left(F_{2} F_{3}\right)$, dont chacune perce le plan $Q$ en $f_{2} f_{3}$ points. Donc à une courbe $f$ correspondent

points $c$.

$$
f_{1} f_{2} f_{3} m_{2} m_{3} m_{r} p_{1} p_{2} p_{3} r^{2}
$$

Il suit de ce que nous avons trouvé quant aux surfaces, que les courbes $\left(F_{2} F_{3}\right)$ remplissent une surface d'ordre

$$
2 f_{2} f_{3} m_{2} m_{3} m_{r} p_{2} p_{3} r
$$

qui rencontre le plan $Q$ en une courbe engendrée par le point $c$ sur $Q$. Ainsi le point $c$ engendre une courbe d'ordre

$$
2 f_{2} f_{3} m_{2} m_{3} m_{r} p_{2} p_{3} r
$$


Par un point arbitraire dans l'espace passent $m_{1}$ surfaces $F_{1}$ et par conséquent de même autant de courbes $f$ passent par un point du plan $Q$. Donc d'ordre $f_{3}$.

par un point quelconque $d u$ plan $Q$ passent $m_{1}$ courbes $f$ qui sont

À l'aide de ce que nous avons démontré dans le paragraphe II nous pouvons dire que la courbe $C_{3}$ est d'ordre

$$
c_{3}=2 f_{1} f_{2} f_{3} m_{1} m_{2} m_{3} m_{r} p_{1} p_{2} p_{3} r^{2}+f_{1} f_{2} f_{3} m_{1} m_{2} m_{3} m_{r} p_{1} p_{2} p_{3} r^{2}
$$

ou

$$
c_{3}=(1+2) m_{r} r^{2} F_{3} M_{3} P_{3}
$$

\section{Sur la courbe qui est le lieu des points d'intersection des surfaces de $n$ faisceaux.}

31. Comme dans nos recherches sur les surfaces nous avons déterminé l'ordre d'une surface $S_{n}$ en connaissant l'ordre d'une surface $S_{n-1}$, nous pouvons appliquer la même voie quant aux courbes.

Soient données:

un faisceau $(R)$ de la $(m-2)^{\text {ième }}$ dimension et d'indice $m_{r}$ de surfaces d'ordre $r$; puis enfin

les courbes $\left(p_{1}\right),\left(p_{2}\right), \ldots\left(p_{n}\right)$ respectivement d'ordre $p_{1}, p_{2}, \ldots p_{n}$; et

les faisceaux $\left(F_{1}\right),\left(F_{2}\right), \ldots\left(F_{n}\right)$ de la première dimension et d'indices $m_{1}, m_{2}, \ldots m_{n}$ de surfaces $F_{1}, F_{2}, \ldots F_{n}$ respectivement d'ordre $f_{1}, f_{2}, \ldots f_{n}$.

Faisons correspondre les surfaces du faisceau $\left(F_{1}\right)$ aux points de la courbe $\left(p_{1}\right)$, etc. Une surface $R$ détermine sur les courbes $\left(p_{1}\right),\left(p_{2}\right), \ldots\left(p_{n}\right)$ des points correspondants. Les surfaces de ces faisceaux passant par les points correspondants sur les courbes $\left(p_{1}\right),\left(p_{2}\right), \ldots\left(p_{n}\right)$ soient appelées de même correspondantes.

32 Prenons une à une de chacun de ces groupes de surfaces correspondantes $F$.

Ces $n$ surfaces, prises trois à trois, se rencontrent en des points. Quand la surface $R$ change de position, il peut arriver que toutes ces $n$ surfaces se rencontrent en un point. On voit très-aisément que le lieu de ces points est une courbe dont l'ordre nous allons déterminer.

Cherchons le nombre de points de rencontre de cette courbe avec un plan arbitraire $Q$. 
Negligeons la courbe $\left(p_{n}\right)$ et son faisceau correspondant $\left(F_{n}\right)$. Nous pouvons dériver une surface $S_{n-1}$ du faisceau $(R)$ et des autres $n-1$ faisceaux $(F)$. La surface $S_{n-1}$ rencontre $?$ en une courbe que nous voulons considérer comme le lieu des points $c$.

En effet nous pouvons obtenir la surface $S_{n-1}$ comme le lieu des courbes d'intersection d'une surface arbitraire $F_{n-1}$ avec la surface correspondante $S_{n-2} ;$ à chacune de ces courbes $\left(F_{n-1} S_{n-2}\right)$ correspond évidemment un certain nombre de surfaces $F_{n}$ du faisceau $\left(F_{n}\right)$, et nous obtenons les points de la courbe $S_{n}$ comme les points de rencontre des courbes $\left(F_{n-1} S_{n-2}\right)$ avec les surfaces correspondantes $F_{n}$ du faisceau $\left(F_{n}\right)$.

Il faut donc que nous connaissions les quantités nécessaires à la détermination de l'ordre de la courbe $S_{n}$.

A un point arbitraire $c$ (qui est proprement dit le point de la courbe $\left.Q S_{n-1}\right)$ correspond une seule surface $R$, de laquelle ce point avait été dérivé. Cette surface $R$ rencontre $\left(p_{n}\right)$ en $r p_{n}$ point et chacun d'eux détermine $m_{n}$ surfaces $F_{n}$ du faisceau $\left(F_{n}\right)$, dont chacune rencontre $Q$ en une courbe $f$. Donc

$\grave{a}$ un point $c$ correspondent $m_{n} p_{n} r$ courbes $f$.

Une surface $F_{n}$ rencontre $Q$ en une courbe $f$ et la courbe $\left(p_{n}\right)$ en $f_{n} p_{n}$ points, dont chacun détermine en $(R)$ un autre faisceau $\left(R^{\prime}\right)$ de la $(n-3)^{\text {ieme }}$ dimension. Nous pouvons dériver de ce faisceau à l'aide des autres faisceaux la courbe $C_{n-1}$ rencontrant le plan $Q$ en les points $c$ correspondant à la courbe $f$. Ainsi

$\grave{a}$ une courbe arbitraire $f$ correspondent $c_{n-1} f_{n} p_{n}$ points $c$. Le lieu de ces points sur $Q$ est une courbe d'ordve $s_{n-1}$. Par un point arbitraire du plan $Q$ passent évidemment $m_{n}$ surfaces $\vec{F}_{n}$ et par conséquent autant de courbes $f$. L'ordre de la courbe f est $f_{n}$.

Cela nous apprend, ayant égard au paragraphe II, que la courbe $C_{n}$ est d'ordre

et puisque

nous obtenons

$$
\begin{gathered}
c_{n}=r f_{n} m_{n} p_{n} s_{n-1}+c_{n-1} f_{n} m_{n} p_{n} \\
s_{n-1}=(n-1) r m_{n} F_{n-1} M_{n-1} P_{n-1}, \\
c_{n}=(n-1) r^{2} m_{r} F_{n} M_{n} P_{n}+c_{n-1} f_{n} m_{n} p_{n} .
\end{gathered}
$$

En traitant la courbe $C_{3}$, nous avons vu que

$$
c_{3}=3 r^{2} m_{2} F_{3} M_{3} P_{3} \text {. }
$$

Nous pouvons écrire le coëfficient 3 sous la forme

$$
\frac{3 \cdot 2}{1 \cdot 2} \text { ou en général } \frac{n(n-1)}{1 \cdot 2} \text {. }
$$


Supposons que pour $C_{n-1}$ la formule

$$
c_{n-1}=\frac{(n-1)(n-2)}{1 \cdot 2} r^{2} m_{r} F_{n-1} M_{n-1} P_{n-1}
$$

est valable, alors pour $C_{n}$ nous obtenons

$$
c_{n}=\frac{n(n-1)}{1 \cdot 2} r^{2} m_{r} F_{n} M_{n} P_{n}
$$

et puisque pour $n=3$ elle est exacte, alors elle est exacte en général; nous avons donc

$$
c_{n}=\frac{n(n-1)}{1 \cdot 2} r^{2} m_{r} \cdot F_{n} M_{n} P_{n}
$$

\section{Cas particuliers.}

33. Courbe du troisième ordre. - En supposant que $n=3$ et toutes les autres quantités sont égales à 1 , nous obtenons une courbe du troisième ordre:

Un plan $R$ du faisceau $(R)$ rencontre les droites $\left(p_{1}\right),\left(p_{2}\right),\left(p_{3}\right)$ en des points, par lesquels passent respectivement les plans $F_{1}, F_{2}, F_{3}$ qui se rencontrent en un point $d$ 'une courbe du troisième ordre.

Nous trouvons que sur un plan du faisceau $\left(F_{1}\right)$ se trouve un seul point ou que l'axe du faisceau $\left(F_{2}\right)$ contient deux points de la courbe; la même propriété jouissent les axes des autres deux faisceaux.

Cette construction de la courbe du troisième ordre est un cas particulier de la génération des surfaces et des courbes due à $\mathrm{M}_{\mathrm{AC}}-\mathrm{L}_{\mathrm{AURIN}}$ que nous avons généralisée ailleurs.

34. Courbe du sixième ordre. - Supposons que $(R)$ soit un faisceau de plans de la deuxième dimension et du premier iudice, ou, en d'autres termes, il est un faisceau de plans déterminés par un seul point $r$. Les lignes $\left(p_{1}\right)$, $\left(p_{2}\right),\left(p_{3}\right),\left(p_{4}\right)$ soient des droites et $\left(F_{1}\right),\left(F_{2}\right),\left(F_{3}\right),\left(F_{4}\right)$ soient des faisceaux de la première dimension et du premier indice; alors ce sont des plans passant par les droites $D_{1}, D_{2}, D_{3}, D_{4}, n$ étant égal à 4 .

Considérons un plan arbitraire $F^{\prime}{ }_{4}$ du fajsceau $\left(F_{4}\right)$ qui rencontre $\left(p_{4}\right)$ en un point $p_{1}^{\prime}$. Ce point détermine avec $r$ un faisceau de plans de la première dimension et du premier indice. Par conséquent, on en peut déduire, à l'aide des droites $\left(p_{1}\right),\left(p_{2}\right),\left(p_{3}\right)$ et des faisceaux $\left(F_{1}\right),\left(F_{2}\right),\left(F_{3}\right)$, une cubique gauche. Cette courbe perce le plan $F^{\prime}{ }_{4}$ en des points qui appartiennent évidemment à 
la courbe $C_{4}$. Puisque nous n'obtenons sur ce plan que ce point, il suit de là que l'axe $D_{4}$ en contient trois.

Les points situés sur $D_{4}$ nous trouvons ainsi: omettons le faicceau $\left(F_{4}\right)$ et la droite $\left(p_{4}\right)$. Du faisceau $(R)$ à l'aide des faisceaux $\left(F_{1}\right),\left(F_{2}\right),\left(F_{3}\right)$ nous pouvons construire une surface $S_{3}$ qui est du troisième ordre.

Elle rencontre $D_{4}$ en trois points qui sont les points de la courbe $C_{4}$. Donc

La courbe $C_{4}$ rencontre chacun des axes des faisceaux $\left(F_{1}\right),\left(F_{2}\right),\left(F_{3}\right)$, $\left(F_{4}\right)$ en trois points.

35. Courbe $C_{5}$ du dixième ordre. - Considérons un faisceau $(R)$ de plans, qui est de la troisième dimension, ou en d'autres termes, dont les plans ne sont pas déterminés et remplissent tout l'espace. De là suit que $n=5$. Cette condition exige que soient données cinq courbes $\left(p_{1}\right), \ldots\left(p_{5}\right)$ et autant de faisceaux $\left(F_{1}\right),\left(F_{2}\right), \ldots\left(F_{5}\right)$ qui sont de la première dimension et du premier indice, c'est-à-dire ce sont des plans passant par cinq droites $D_{1}, D_{2}, D_{3}, D_{4}, D_{3}$.

Un plan quelconque $F_{5}^{\prime}$ du faisceau $\left(F_{5}\right)$ rencontre $\left(p_{5}\right)$ en le point $p_{5}^{\prime}$ qui détermine un faisceau de plans de la seconde dimension duquel on peut dériver à l'aide des faisceaux $\left(F_{1}\right),\left(F_{2}\right),\left(F_{8}\right),\left(F_{4}\right)$ et des droites $\left(p_{1}\right),\left(p_{2}\right)$, $\left(p_{3}\right),\left(p_{4}\right)$ une courbe du sixième ordre (l'article précédent). Cette courbe rencontre le plan $F_{5}^{\prime}$ en six points d'une courbe $C_{5}$ du dixième ordre qui coupe, par conséquent, chacun des axes des faisceaux $(F)$ en quatre points. Ainsi:

points; et

Chacun des axes de cinq faisceaux ( $F$ ) rencontre la courbe $C_{5}$ en quatre six points.

un plan arbitraire d'un des faisceaux $(F)$ coupe cette courbe encore en

Les points de rencontre des axes de ces faisceaux avec la courbe $C_{5}$ peuvent être déterminés comme il suit.

En chcrchant les points $c_{5}$ sur $D_{5}$, nous faisons usage seulement de quatre faisceaux $\left(l^{\top}\right)$ et de quatre courbes $(p)$ qui déterminent avec un faisceau de plans de la troisième dimension une surface $S_{3}$ du quatrième ordre. Cette surface rencontre la droite $D_{5}$ en des points de la courbe $C_{5}$; de même pour les autres axes $D_{1}, D_{2}, D_{3}, D_{4}$ des faisceaux $(F)$.

36. Autres cas particuliers. - Achevons en peu de mots encore quelques autres cas particuliers de la construction des courbes.

Supposons que le faisceau $(R)$ de plans soit de la première dimension et du premier indice et que tous les trois faisceaux $(F)$ de surfaces du second ordre soient de même de la première dimension et d'indice 1 , et enfin que les lignes $(p)$ soient des droites. 
Alors la courbe $C_{3}$ est d'ordre

$$
c_{3}=3 \cdot 2^{3}=24 \text {. }
$$

Une surface arbitraire $F_{3}^{\prime}$ rencontre la droite $\left(p_{3}\right)$ en deux points, dont chacun détermine un plan rencontrant $\left(p_{1}\right),\left(p_{2}\right)$ en des points; chacun d'eux détermine une surface du second ordre; ces surfaces se rencontrent en une biquadratique gauche qui perce $F_{3}^{\prime}$ en huit points. Nous obtenons ainsi seize points directement.

En négligeant le faisceau $\left(F_{3}\right)$, nous obtenons une surface $S_{2}$ du huitième ordre qui rencontre la courbe fondamentale du faisceau $\left(F_{3}\right)$ en 32 points qui appartiennent évidemment à la courbe $C_{3}$.

D'où il suit que

La courbe $C_{3}$ possède 32 points sur chaque courbe fondamentale des faisceaux $(F)$.

37. Les courbes du $24^{\text {ìme }}$ ordre résultent aussi des faisceaux de plans quand les courbes $(p)$ sont des coniques.

Un plan quelconque $F_{3}$ rencontre $\left(p_{3}\right)$ en deux points dont chacun détermine un plan $R$ et chacun de ces plans coupe les coniques $\left(p_{1}\right),\left(p_{2}\right)$ en deux à deux points. De là résulte que nous obtenons de chacun des plans $R$ quatre droites $F_{1} F_{2}$ qui percent $F_{3}$ en des points de la courbe $C_{3}$. Nous obtenons alors sur chaque plan $\left(F_{3}\right)$ huit points directement. En omettant le faisceau $\left(F_{3}\right)$ et la conique $\left(p_{3}\right)$, le faisceau $(R)$ détermine une surface $S_{2} d u$ huitième ordre. Cette surface rencontre $D_{1}$ en huit points qui sont les points doubles, ce qui prouve le raisonnement suivant.

A un de ces points correspond un seul plan $R$ qui rencontre $\left(p_{3}\right)$ en deux points et chacun d'eux détermine un plan $F_{3}$. Ces deux plans ainsi obtenus fournissent le même point. Donc

La courbe $C_{3}$ est du $24^{\text {ième }}$ ordre douée des 24 points doubles qui sont situés huit à huit sur trois droites.

38. Considérons encore le cas quand $n=3$, toutes les lignes $(p)$ sont des droites, $(R)$ est un faisceau de plans de la première dimension et d'indice 1 , et enfin $(F)$ sont les faisceaux de la première dimension de surfaces d'ordre $f$.

La courbe $C_{3}$ est done d'ordre

Ainsi :

$$
c_{3}=3 f^{3}
$$

Sur une surface quelconque d'un faisceau se trouvent $f^{4}$ points. Chacunes des courbes fondamentales des faisceaux contient $2 f^{4}$ points de la courbe $C_{z}$. 


\section{Décomposition des figures dérivées.}

39. La surface dérivée se décompose, quand un point fondamental $a \mathrm{du}$ faisceau $\left(F_{n}\right)$ se trouve sur $\left(p_{n}\right)$.

Considérons la position de la surface $R$, quand elle passe par ce point $a$. Ce point détermine dans le faisceau $(R)$ un autre faisceau de la $(n-2)^{\text {ième }}$ dimension, qui avec les autres $(n-1)$ faisceaux, excepté $\left(F_{n}\right)$, détermine une surface $S_{n-1}$. Quand nous voulons déterminer la surface $F_{n}$, nous trouvons qu'elle est indéterminée, parce que par ce point passe une infinité de surfaces du faisceau $\left(F_{n}\right)$.

Nous voyons donc que la surface $S_{n-2}$ fait une partie de la surface $S_{n}$. D'où il suit que

Un faisceau, ayant un de ses points fondamentaux sur la courbe correspondante, ce point détermine dans le faisceau $(R)$ un autre faisceau d'une dimension inférieure, dont la surface dérivée à l'aide des autres $(n-1)$ faisceaux fait une partie de la surface $S_{n}$.

40. La surface $S_{n}$ se décompose, quand la courbe fondamentale du faisceau $(R)$ rencontre $\left(p_{n}\right)$ en un point $a$.

Ce point détermine $m_{n}$ surfaces $F_{n}$ du faisceau $\left(F_{n}\right)$. Considérons une de ces surfaces et un de ses points $f$. Nous pouvons démontrer que ce point appartient à la surface $S_{n}$.

Ce point $f$ détermine dans chacun des antres $n-1$ faisceaux plusieurs surfaces, dont chacune rencontre la courbe correspondante en des points. En prenant un à un point de ces groupes, nous obtenons $n-1$ points qui déterminent des surfaces $R$, dont chacune rencontre $\left(p_{n}\right)$ au point a et par conséquent la surface $F_{n}$ passant par $a$ correspond aux surfaces des autres faisceaux, qui passe par le point $f$.

Puisque par le point $f$ passent $n$ surfaces correspondantes et ce point, étant pris à volonté sur la surface $F_{n}$ qui passe par $a$, nous pouvons donc dire que

Quand un point fondamental du faisceau $(R)$ se trouve sur la courbe $\left(p_{n}\right)$, les surfaces du faisceau $\left(F_{n}\right)$, qui passent par ce point forment les parties de la surface générale de $S_{n}$.

41. Nous allons démontrer que la même chose a lieu quant aux courbes.

Le faisceau $\left(F_{n}\right)$ a un de ses points fondamentaux a sur la courbe correspondante $\left(p_{n}\right)$. 
Le point a détermine dans le faisceau $(R)$ un nouveau faisceau de la

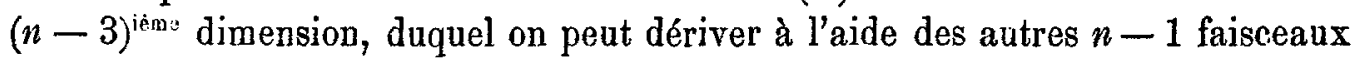
une courbe $C_{n-1}$. Cette courbe fait évidemment une partie de la courbe $C_{n}$, parce que chaque surface $F_{n}$ correspond aux surfaces qui engendrent la courbe $C_{n-1}$. Par conséquent chaque point de la courbe $C_{n-1}$ appartient à la courbe $C_{n}$.

Done

Quand un faisceau $(F)$ a un de ses points fondamentaux sur la courbe correspondante $(p)$, ce point détermine dans le faisceau $(R)$ un nouveau faisceau de la dimension inférieure et la courbe $C_{n-1}$, dérivée de ce faisceau à l'aide des autres fuisceaux de surfaces, fait une partie de la courbe générale $C_{n}$.

42. La courbe $\left(p_{n}\right)$ passe par un point fondamental du faisceau $(R)$ de la $n^{\text {ième }}$ dimension.

Le faisceau $(R)$ de la $(n-2)^{\text {ième }}$ dimension détermine à l'aide des autres $n-1$ faisceaux une surface $S_{n-1}$ qui rencontre les surfaces du faisceau $\left(F_{n}\right)$, passant par le point $a$, en des courbes qui sont évidemment les parties de la courbe $C_{n}$.

Nous avons donc ce théorème:

Quand un point fondamental du faisceau $(R)$ est situé sur une des courbes $(p)$, alors les courbes d'intersection des surfaces du faisceau correspondant à $(p)$, qui passent par ce point, avec la surface dérivée du faisceau $(R) \grave{a}$ l'aide des autres faisceaux, font les parties de la courbe $C_{n}$.

\section{L'ordre de la surface dégénérée.}

43. Supposons que les courbes fondamentales des faisceaux $\left(F_{1}\right),\left(F_{2}\right), \ldots$ $\left(F_{n}\right)$ rencontrent leurs courbes correspondantes $\left(p_{1}\right),\left(p_{2}\right),\left(p_{3}\right), \ldots\left(p_{n}\right)$ en les points $F_{1}, F_{2}, \ldots F_{n}$, puis que la courbe fondamentale du faisceau $(R)$ coupe les mêmes courbes en des points $R_{1}, R_{2}, \ldots R_{n}$.

Quand nous voulons déterminer l'ordre de la surface $S_{n}$, nous suivons la même voie comme dans le cas général et soustrayons alors les dits points fondamentaux du nombre général.

44. Appliquons de nouveau une droite $Q$. En prenant un point arbitraire $p_{n}^{\prime}$ sur $\left(p_{n}\right)$, ce point détermine dans le faisceau $(R)$ un autre faisceau de la $(n-2)^{\text {irmè }}$ dimension, duquel nous pouvons dériver une surface $S_{n-1}$ à l'aide des autres $(n-1)$ faisceaux et des courbes. Cette surface rencontre $Q$ en les points $B$ et les surfaces $F_{n}$ du faisceau $\left(F_{n}\right)$, qui passent par le point $\boldsymbol{p}_{n}^{\prime}$, coupent la droite $Q$ en les points $A$ correspondant aux points $B$. 
Considérons un point quelconque de la droite $Q$ comme le point $B$.

Par ce point passent $m_{1}$ surfaces $F_{1}$ du faisceau $\left(F_{1}\right)$, dont chacune rencontre la courbe $\left(p_{1}\right)$ outre les dits points fondamentaux encore en $f_{1} p_{1}-F_{1}$ points. Ce point $B$ détermine de même $m_{2}, m_{3}, \ldots m_{n-1}$ surfaces $F_{2}, F_{3}, \ldots F_{n-1}$ des faisceaux $\left(F_{1}\right),\left(F_{2}\right), \ldots\left(F_{n-1}\right)$, et ces surfaces rencontrent les courbes correspondantes $\left(p_{2}\right),\left(p_{3}\right), \ldots\left(p_{n-1}\right)$ outre les points fondamentaux encore en

$$
f_{2} p_{2}-F_{2}, \quad f_{3} p_{3}-F_{3}, \ldots \quad f_{n-1} p_{n-1}-F_{n-1}
$$

points qui, étant pris un à un sur chaque courbe, déterminent chaque fois $m_{r}$ surfaces $R$. Ces points déterminent en entier

surfaces $R$.

$$
m_{r} M_{n-1}\left(f_{1} p_{1}-F_{1}\right)\left(f_{2} p_{2}-F_{2}\right) \cdots\left(f_{n-1} p_{n-1}-F_{n-1}\right)
$$

Pour abréger, écrivons au lieu de terme

le symbole

$$
\left(f_{1} p_{1}-F_{1}\right)\left(f_{2} p_{2}-F_{2}\right) \cdots\left(f_{n-1} p_{n-1}-F_{n-1}\right)
$$

$$
\Pi_{n-1}(f p-F)
$$

la signification de $\Pi_{n_{-1}}$ est assez claire, car il remplace l'expression " le produit n et son index signifie qu'il faut poser dans les parenthèses en même temps le même index; la valeur de cet index varie de 1 à $n-1$; les binomes ainsi construits sont les facteurs de ce produit.

Nous arons donc:

$$
\Pi_{n-1}(f p-F)=\left(f_{1} p_{1}-F_{1}\right)\left(f_{2} p_{2}-F_{2}\right) \cdots\left(f_{n-1} p_{n-1}-F_{n-1}\right) .
$$

Chacune des surfaces ainsi obtenues détermine sur $\left(p_{n}\right)$ outre les points fondamentaux encore $\left(r p_{n}-R_{n}\right)$ points et chacun d'eux détermine $m_{n}$ surfaces $F_{n}$, dont chacune rencontre $Q$ en $f_{n}$ point $A$ correspondant au point $B$ pris à volonté sur $Q$.

$A$ un point $B$ correspondent donc

points $A$.

$$
m_{r} M_{n} f_{n}\left(r p_{n}-R_{n}\right) \Pi_{n-1}(f p-F)
$$

Considérons maintenant un point arbitraire de $Q$ comme le point $A$. Par ce point passent $m_{n}$ surfaces $F_{n}$ du faisceau $\left(F_{n}\right)$ dont chacune rencontre la courbe $\left(p_{n}\right)$ outre les dits points fondamentaux encore en $f_{n} p_{n}-F_{n}$ points, et chacun d'eux détermine un faisceau $(R)$ de la $(n-2)^{\text {ipme }}$ dimension. De chacun de ces faisceaux $(R)$ nous pouvons dériver à l'aide des autres $(n-1)$ faisceaux $(F)$ une surface $S_{n-1}$ qui rencontre la droite $Q$ en $s_{n-2}$ points $B$ correspondant au point $A$. 
A. un point $A$ correspondent donc

points $B$.

$$
m_{n}\left(f_{n} p_{n}-F_{n}\right) s_{n-1}
$$

La courbe $S_{n}$ est alors d'ordre

$$
s_{n}=m_{n}\left(f_{n} p_{n}-F_{n}\right) s_{n-1}+m_{v} f_{n} M_{n}\left(r p_{n}-R_{n}\right) \Pi_{n-1}(f p-F) .
$$

On voit très aisément que pour $n=2$ la surface $S_{n-1}$ se compose de plusieurs surfaces $F_{1}$ et qu'il faut poser dans la formule précédente pour $s_{1}$ le terme

$$
f_{1} m_{1} m_{r}\left(p_{1} r-R_{1}\right)
$$

la formule prend la forme

$$
s_{2}=m_{2}\left(f_{2} p_{2}-F_{2}\right) f_{1} m_{1} m_{r}\left(p_{1} r-R_{1}\right)+m_{r} m_{1} m_{2} f_{2}\left(r p_{2}-R_{2}\right)\left(f_{1} p_{1}-F_{1}\right),
$$

ce que nous pouvons écrire ainsi:

$$
s_{2}=m_{r} M_{2}\left(f_{1} \frac{p_{1} r-R_{1}}{f_{1} p_{1}-F_{1}}+f_{2} \frac{p_{2} r-R_{2}}{f_{2} p_{2}-F_{2}}\right) \Pi_{2}(f p-F) .
$$

Dans ce qui va suivre nous allons écriver

au lieu de

$$
\sum_{i=1}^{2}\left(f_{i} \frac{r p_{i}-R_{i}}{f_{i} p_{i}-F_{i}^{\prime}}\right)
$$

et en général

$$
f_{1} \frac{r p_{1}-R_{1}}{f_{1} p_{1}-F_{1}}+f_{2} \frac{r p_{2}-R_{2}}{f_{2} p_{2}-I_{2}}
$$

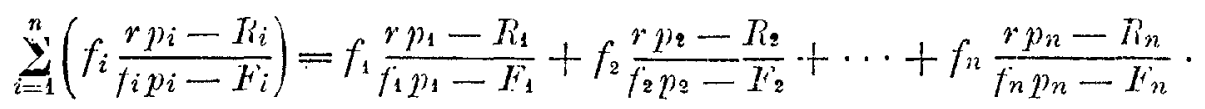

La signification de ce symbole est assez claire.

Acceptons que pour $n-1$ la formule

$$
s_{n-1}=m_{r} M_{n-1} \Pi_{n-1}(f p-F) \sum_{i=1}^{n-1}\left(f_{i} \frac{r^{\prime} p_{i}-R_{i}}{f_{i} p_{i}-F_{i}}\right)
$$

soit exacte, alors la formule pour $n$ prend la forme

et puis que

$$
\begin{gathered}
s_{n}=m_{n}\left(f_{n} p_{n}-F_{n}\right) m_{r} M_{n-1} \Pi_{n-1}\left(f p-F^{r}\right) \sum_{i=1}^{n-1} f_{i} \frac{p_{i} r-R_{i}}{f_{i} p_{i}-I_{i}} \\
+m_{r} M_{n} f_{n}\left(r p_{n}-R_{n}\right) \Pi_{n-1}\left(f p-F^{\prime}\right)
\end{gathered}
$$

$$
\left(f_{n} p_{n}-F_{n}\right) \Pi_{n-1}\left(f p-F^{\prime}\right)=\Pi_{n}\left(f p-F^{\prime}\right)
$$


nous obtenons

Tandis que

$$
s_{n}=m_{r} M_{n} \Pi_{n}(f p-F)\left[\sum_{i=1}^{n-1}\left(f_{i} \frac{r p_{i}-R_{i}}{f_{i p_{i}}-F_{i}}\right)+f_{n} \frac{r p_{n}-R_{n}}{f_{n} p_{n}-F_{n}}\right] .
$$

$$
\sum_{i=1}^{n-1}\left(f_{i} \frac{r p_{i}-R_{i}}{f_{i p_{i}}-F_{1}}\right)+f_{n} \frac{r p_{n}-R_{n}}{f_{n} p_{n}-F_{n}}=\sum_{i=1}^{n}\left(f_{i} \frac{r p_{i}-R_{i}}{f_{i} p_{i}-F_{i}}\right)
$$

nous pouvons écrire

$$
s_{n}=m_{s} M_{n} \Pi_{n}(f p-F) \sum_{i=1}^{n}\left(f_{i} \frac{p_{i} r-R_{i}}{f_{i} p_{i}-F_{i}}\right) .
$$

Nous avons ainsi démontré que cette formule est exacte si elle est valable pour $n-1$. Tandis que elle est valable pour $n=2$ et par conséquent pour $n=3$ et si suit, alors cette formule est exacte en général.

De là suit que la courbe propre $S_{n}$ est d'ordre

$$
s_{n}=m_{r} M_{n} \Pi_{n}(f p-F) \sum_{i=1}^{n}\left(f_{i} \frac{r p_{i}-P_{i}}{f_{i} p_{i}-F_{i}}\right) .
$$

45. Quoique cette formule exprime l'ordre de la surface $S_{n}$ quand les données occupent des positions singulières, elles est, on le voit, plus générale que la formule de l'article 19, qui peut être déduite de la formule actuelle.

Supposons que le nombre de points fondamentaux sur $(p)$ soit

et de même

$$
F_{1}=F_{2}=\cdots-F_{n}-\theta
$$

nous obtenons

$$
R_{1}=R_{2}=\cdots=R_{n}=0 \text {, }
$$

et

$$
f p-F=f p
$$

D'oì il suit que

$$
f_{i} \frac{p_{i} r-R_{i}}{f_{i} p_{i}-I_{i}}=f_{i} \frac{p_{i} r}{p_{i} f_{i}}=r
$$

$$
\sum_{i=1}^{n}\left(f_{i} \frac{p_{i} r-R_{i}}{f_{i p_{i}-F_{i}}}\right)=n r
$$

et la formule $(a)$ prend la forme

$$
s_{n}=m_{r} n r M_{n} \Pi_{n}(f p)
$$

ce risultat est done identique avec celui de l'article 19.

46. De la formule $(a)$ on peut dériver beaucoup d'autres, dont unes sont particulières, et les autres paraissent d'être générales et tout entier différentes. 


\section{Constructions générales des surfaces déduites du précédent.}

47. Premier cas. - Considérons d'abord la construction d'une surface quand les surfaces $F$ sont des plans et par conséquent les fajsceaux $\left(F_{1}\right)$, $\left(F_{2}\right), \ldots\left(F_{n}\right)$ sont des surfaces développables respectivement de la classe $m_{1}$, $m_{2}, \ldots m_{n}$.

Cependant il faut distinguer ces deux cas: $a$ ) où $(R)$ est un faisceau de surfaces générales, $b$ ) où $(R)$ est un faisceau de plans.

a) Supposons que les surfaces du faisceau $(R)$ soient d'ordre $r$. La surface obtenue $S_{n}$ est d'ordre

$$
m_{r} M_{n} \Pi_{n}(p-F) \sum_{i=1}^{n}\left(\frac{p_{i} r-R_{i}}{p_{i}-F_{i}}\right)
$$

$F$ étant en général $=0$. La construction d'un point de la surface est la suivante:

Un faisceau $(R)$ de la dimension $(n-1)$ de surfaces $R$ d'ordre $r$ rencontre les courbes $\left(p_{1}\right),\left(p_{2}\right), \ldots\left(p_{n}\right)$ en des points par lesquels passent les plans tangents des surfaces développables $\left(F_{1}\right),\left(F_{2}\right), \ldots\left(F_{n}\right)$ de la classe $m_{1}, m_{2}, \ldots$ $m_{n}$; quand tous les plans ainsi correspondants passent par un même point, ce point appartient $\grave{a}$ la surface $S_{n}$.

48. Le cas, dont nous venons de parler, renferme aussi tel, quand les surfaces développables deviennent des surfaces coniques qui ont leurs sommets respectivement sur les courbes correspondantes. Ainsi:

Soient données $n$ surfaces coniques $\left(F_{1}\right),\left(F_{2}\right), \ldots\left(F_{n}\right)$ de la classe $m_{1}$, $m_{2}, \ldots m_{n}$, dont les sommets se trouvent respectivement sur les courbes correspondantes $\left(p_{1}\right),\left(p_{2}\right), \ldots\left(p_{n}\right)$.

Quand $n$ plans tangents de ces surfaces passent par un point s de telle manière qu'ils rencontrent les courbes $\left(p_{1}\right),\left(p_{2}\right), \ldots\left(p_{n}\right)$ en des points qui, étant pris un à un sur chacune de ces courbes, déterminent une surface $R$ d'un faisceau de la dimension $n-1$, ce point $s$ appartient à une surface d'ordre

$$
m_{r} M_{n} \Pi_{n}(p-1) \sum_{i=1}^{n}\left(\frac{p_{i} r-R_{i}}{p_{i}-1}\right) \text {. }
$$

Nous obtenons le cas encore plus particulier, quand les lignes fondamentales des faisceaux $(F)$ sont des droites.

b) Considérons le cas, quand les faisceaux $(F)$ sont des surfaces développables et $R$ sont des plans. 
Nous pouvons distinguer trois cas particuliers, savoir:

1. ${ }^{\circ}$ le faisceau $(R)$ de plans $R$ est de la première dimension;

2. le même faisceau est do la deuxième dimension.

Nous avons traités ces deux cas dans les articles 25 et 26.

$3 .^{\circ}$ Le faisceau $(R)$ de plans $R$ est de la troisième dimension, ou, en d'autres termes, les plans $R$ ne sont assujettis ni à une condition.

La construction d'un point $s$ de la surface peut être énoncée ainsi:

Soient données quatre surfaces développables $\left(F_{1}\right),\left(F_{2}\right),\left(F_{3}\right),\left(F_{4}\right)$ respectivement de la classe $n_{1}, m_{2}, m_{3}, m_{1}$. Par un point quelconque $s$ dans l'espace passent les plans tangents à ces surfaces, qui rencontrent les courbes correspondantes $\left(p_{1}\right),\left(p_{2}\right),\left(p_{3}\right),\left(p_{4}\right)$ en des points. Quand quatre de ces points, étant pris un à un sur chaque courbe $(p)$, se trouvent sur un plan, le point $s$ appartient à une surface d'ordre

$$
m_{r}, M_{4} \Pi_{4}(p-F) \sum_{i=1}^{4}\left(\frac{p_{i}}{p_{i}-F_{i}}\right) .
$$

On voit que $F$ ne doit pas surpasser 2 .

49. Deuxième cas. - Supposons que $(F)$ soient des faisceaux de surfaces générales et que

a) $(R)$ soit un faisceau de plans.

Nous pouvons distinguer de nouveau les cas suivants:

1. ${ }^{\circ}(R)$ est un faisceau de la première dimension, ou il forme une surface développable. Dans ce cas, les faisceaux $(F)$ peuvent être seulement deux, et nous obtenons cette construction:

Les plans tangents d'une surface développable de la classe $m_{r}$ rencontrent les courbes $\left(p_{1}\right),\left(p_{2}\right)$ en des points qui déterminent des surfaces $F_{1}$, $F_{2}$ dans les faisceaux $\left(F_{1}\right),\left(F_{2}\right)$ correspondants. Les surfaces $F_{1}, F_{2}$ correspondantes se coupent en une courbe qui engendre une surface d'ordre

$$
m_{r} M_{2} \Pi_{2}(f p-F) \sum_{i=1}^{2}\left(f_{i} \frac{p_{i}-R_{i}}{f_{i} p_{i}-F_{1}}\right) .
$$

C'est évidemment un cas particulier de la surface que nous avons traitée au commencement de ce Mémoire.

2. Nous pourrions de même donner la construction d'une surface, quand le plan $R$ enroloppe une surface générale de la classe $m_{r}$.

$3 .^{\circ}$ Quand le faisceau $(R)$ est de la troisième dimension, nous trouvons la construction donnée dans l'article $48,3^{\circ}$. 
b) Le cas que nous allons traiter nous semble très-important. Supposons que tous les faisceaux sont de la première dimension, c'est-à-dire

et que

$$
m_{1}=m_{2}=\cdots=m_{n}=m_{r}=1
$$

$$
f_{i} p_{i}-F_{i}=1, \quad p_{i} r-R_{i}=k,
$$

$k$ étant une grandeur constante.

L'ordre de la surface peut être donnée en cette forme

En supposant que

$$
s_{n}=k \sum_{i=1}^{n} f_{i}
$$

nous obtenons

$$
p_{i} r-R_{i}=k=1
$$

$$
s_{n}=\sum_{i=1}^{n} f_{i}=f_{1}+f_{2}+f_{3}+\cdots+\cdot f_{n} .
$$

On voit immédiatement que l'ordre de la surface $S_{n}$ ne dépend pas d'ordre des surfaces $R$ et des courbes $(p)$; les faisceaux $(F)$ jouissent de telle proprièté que, en prenant une à une surface des $n-1$ faisceaux, à ces surfaces correspond une seule surface du $n^{i e m e}$ faisceau.

Nous pourons énoncer ce théorème:

Soient donnés $n$ faisceaux $\left(F_{1}\right),\left(F_{2}\right), \ldots\left(F_{n}\right)$ de la première dimension de surfaces $F_{1}, F_{2}, \ldots F_{n}$ respectivement d'ordre $f_{1} \cdot f_{2}, \ldots f_{n}$. Faisons correspondre les surfaces des faisceaux de telle manière que, en prenant une $\grave{a}$ une surface dans $n-1$ faisceaux, à ces surfaces correspond une seule surface du $n^{\text {iene }}$ faisceau.

Il y a une infinité de points en lesquels se rencontrent toutes les $n$ surfaces correspondantes, et ces points remplissent une surface dont l'ordre est égal à la somme des ordres des surfaces de tous les faisceaux.

\section{Décomposition d'une courbe.}

50. La décomposition d'une courbe dépend de mêmes conditions que nous avons trouvées en traitant les surfaces.

1. Quand le faisceau $(R)$ a un de ses points fondamentaux a sur la courbe $\left(p_{n}\right)$, nous pouvons de ce faisceau dériver à l'aide des autres $n-1$ faisceaux une surface $S_{n-1}$. 
Le point $a$ détermine dans le faisceau $\left(F_{n}\right) m_{n}$ surfaces $F_{n}$ qui rencontrent la surface, dont nous venons de parler, en des courbes qui appartiennent évidemment à la courbe $C_{n}$ et forment par conséquent une partie de cette courbe.

2. Supposons qu'un point fondamental $a$ du faisceau $\left(F_{n}\right)$ se trouve sur la courbe $\left(p_{n}\right)$. Ce point détermine dans le faisceau $(R)$ un autre faisceau de la $(n-3)^{\text {iemo }}$ dimension, duquel nous pouvons construire à l'aide des autres $(n-1)$ faisceaux $(F)$ une courbe $C_{n-1}$ qui est une partie de la courbe demandée $O_{n}$.

\section{L'ordre de la courbe dégénérée.}

51. Soient $F_{1}, F_{2}, \ldots F_{n}$ les nombres de points d'intersection des courbes fondamentales des faisceaux $\left(F_{1}\right),\left(F_{2}\right), \ldots\left(F_{n}\right)$ avec les courbes correspondantes $\left(p_{1}\right),\left(p_{2}\right), \ldots\left(p_{n}\right)$ et $R_{1}, R_{2}, R_{n}$ soient les nombres de points fondamentaux du faisceau $(R)$ situés sur les mêmes courbes.

Considérons un plan quelconque $Q$ et désignons par $c$ les points, en lesquelles la courbe $S_{n-1}$ perce ce plan $Q$, et par $f$ les courbes, en lesquelles le rencontrent les surfaces $F_{n}$.

A un point $c$ correspond une seule surface $R$ qui rencontre la courbe $\left(p_{n}\right)$ outre les points fondamentaux encore en $\left(p_{n} r-R_{n}\right)$ points, dont chacun détermine $m_{n}$ surfaces $\left(F_{n}\right)$ rencontrant $Q$ en des courbes $f$ qui correspondent au point $c$. A un point $c$ correspondent donc

courbes $f$.

$$
m_{n}\left(p_{n} r-R_{n}\right)
$$

Une surface quelconque $F_{n}$ du faisceau $\left(F_{n}^{\prime}\right)$ détermine une courbe $f$ et rencontre $\left(p_{n}\right)$ outre les points fondamentaux encore en $\left(p_{n} f_{n}-F_{n}\right)$ points.

Chacun d'eux détermine un faisceau $(R)$ de la dimension $(n-3)$, duquel on peut à l'aide des autres $n-1$ faisceaux dériver une courbe $C_{n-1}$ qui détermine les points $c$ correspondant à la courbe $f$ primitive.

Done

$A$ une courbe $f$ correspondent

$$
\left(f_{n} p_{n}-F_{n}\right) c_{n-1}^{\prime}
$$

points $c$. Le point $c$ engendre sur $Q$ une courbe $S_{n-4}$. Par un point arbitraire du plan $Q$ passent $m_{n}$ surfaces $F_{n}$ et par conséquent autant de courbes $f$. La courbe $f$ est d'ordre $f_{n}$. L'ordre de la courbe $C_{n}$ est donc

$$
c_{n}=f_{n} m_{n} s_{n-1}\left(p_{n} r-R_{n}\right)+m_{n}\left(f_{n} p_{n}-F_{n}\right) c_{n-1}^{\prime} .
$$


Nous voyons que pour $n=3$ à un point $c$ correspondent

$$
m_{3}\left(p_{3} r-R_{3}\right)
$$

courbes $f$. $\mathbf{A}$ une courbe $f$ correspondent

$$
m_{r} M_{2} f_{1} f_{2}\left(p_{3} f_{3}-F_{3}\right)\left(r p_{1}-R_{1}\right)\left(r p_{2}-R_{2}\right)
$$

points $c$. Ces points engendrent une courbe d'ordre

$$
m_{r} M_{2} \Pi_{2}(f p-F) \sum_{i=1}^{2}\left(f_{i} \frac{p_{i} r-R_{i}}{f_{i} p_{i}-F_{i}}\right) \text {. }
$$

Par un point arbitraire du plan $Q$ passent $m_{3}$ courbes $f$ d'ordre $f_{3}$. De là suit que

$$
\begin{aligned}
c_{3} & =f_{3}\left(p_{3} r-R_{3}\right) m_{r} M_{2} \Pi_{2}(f p-F) \sum_{i=1}^{2}\left(f_{i} \frac{p_{i} r-R_{i}}{f_{i} p_{i}-F_{i}}\right) \\
& +m_{r} M_{3} f_{1} f_{2}\left(p_{3} f_{3}-F_{3}\right)\left(r p_{1}-R_{1}\right)\left(r p_{2}-R_{2}\right),
\end{aligned}
$$

ce que nous pouvons écrire ainsi:

$$
\begin{gathered}
c_{3}=m_{r} M_{3} \Pi_{3}(f p-F)\left[f_{3} \frac{p_{3} r-R_{3}}{f_{3} p_{3}-F_{3}} \sum_{i=1}^{2}\left(f_{i} \frac{p_{i} r-R_{i}}{f_{i} p_{i}-F_{i}}\right)\right. \\
\left.+f_{1} f_{2} \frac{\left(p_{1} r-R_{1}\right)\left(p_{2} r-R_{2}\right)}{\left(f_{1} p_{1}-F_{1}\right)\left(f_{2} p_{2}-F_{2}\right)}\right] .
\end{gathered}
$$

Nous pouvons remplacer le terme

$$
f_{3} \frac{\left(p_{3} r-R_{3}\right)^{\prime}}{\left(f_{3} p_{3}-F_{3}\right)} \sum_{i=1}^{2}\left(f_{i} \frac{p_{i} r-R_{i}}{f_{i} p_{i}-F_{i}}\right)+f_{1} f_{2} \frac{\left(p_{1} r-R_{1}\right)\left(p_{2} r-R_{2}\right)}{\left(f_{1} p_{1}-F_{1}\right)\left(f_{2} p_{2}-F_{2}\right)}
$$

par le suivant

$$
\sum_{i \Xi t}^{1_{\Xi} 3}\left[f_{i} f_{t} \frac{\left(p_{i} r-R_{i}\right)\left(p_{t} r-R_{t}\right)}{\left(f_{i} p_{i}-F_{i}\right)\left(f_{t} p_{t}-F_{t}\right)}\right]
$$

Le symbole $\sum_{i \leqq t}^{1.3}$ signifie que dans les parenthèses il faut poser pour $i$ et $t$ toutes valeurs possibles de 1 à 3 de telle manière, que toutes les deux ne soient pas égales en même temps, et que résultats ainsi obtenus doivent être additionés.

Ainsi la formule, qui exprime l'ordre de la courbe $C_{3}$, prend la forme suivante:

$$
c_{3}=m_{r} M_{3} \Pi_{3}\left(f p-F^{r}\right) \sum_{i=t}^{1_{1}^{3}}\left[f_{i} f_{t} \frac{\left(p_{i} r-R_{i}\right)\left(p_{t} r-R_{t}\right)}{\left(f_{i} p_{i}-F_{i}\right)\left(f_{t} p_{t}-F_{t}\right)}\right]
$$

Supposons que la formule

$$
c_{n-1}=m_{r} M_{n-1} \Pi_{n-1}(f p-F) \sum_{i \geqq t}^{1,(n-1)}\left[f_{i} f_{t} \frac{\left(p_{i} r-R_{i}\right)\left(p_{t} r-R_{t}\right)}{\left(f_{i} p_{i}-F_{i}\right)\left(f_{t} p_{t}-F_{t}\right)}\right]
$$


pour $n-1$ soit exacte; nous obtenons pour $n$

$$
\begin{gathered}
c_{n}=m_{n} f_{n}\left(p_{n} r-R_{n}\right) m_{r} M_{n-1} \Pi_{n-1}(f p-F) \sum_{i}^{n-1}\left(f_{i} \frac{p_{i} r-R_{i}}{f_{i} p_{i}-F_{i}}\right) \\
+m_{n}\left(f_{n} p_{n}-F_{n}\right) m_{r} M_{n-1} \Pi_{n-1}(f p-F) \sum_{i \leqq t}^{n-1}\left[f_{i} f_{t} \frac{\left(p_{i} r-R_{i}\right)\left(p_{t} r-R_{t}\right)}{\left(f_{i} p_{i}-F_{i}\right)\left(f_{t} p_{t}-F_{t}\right)}\right]
\end{gathered}
$$

ce que nous pouvons écrire ainsi:

$$
\begin{aligned}
c_{n}=m_{r} & M_{n} \Pi_{n}(f p-F)\left[f_{n} \frac{\left(p_{n} r-R_{n}\right)}{\left.f_{n} p_{n}-F_{n}\right)} \sum_{i}^{n-1} f_{i} \frac{p_{i} r-R_{i}}{f_{i p_{i}}-F_{i}}\right. \\
& \left.+\sum_{i \supseteq t}^{n-1}\left(f_{i} f_{t} \frac{\left(p_{i} r-R_{i}\right)\left(p_{t} r-R_{t}\right)}{\left(f_{i} p_{i}-F_{i}\right)\left(f_{t} p_{t}-F_{t}\right)}\right)\right] .
\end{aligned}
$$

Il est visible que

$$
\begin{gathered}
f_{n} \frac{\left(p_{n} r-R_{n}\right)}{\left(f_{n} p_{n}-F_{n}\right)} \sum_{i}^{n-1} f_{i} \frac{\left(p_{i} r-R_{i}\right)}{\left(f_{i} p_{i}-F_{i}\right)}+\sum_{i \triangleq t}^{n-1}\left[f_{i} f_{t} \frac{\left(p_{i} r-R_{i}\right)\left(p_{t} r-R_{t}\right)}{\left(\tilde{f}_{i} p_{i}-F_{i}\right)\left(f_{t} p_{t}-F_{t}\right)}\right] \\
=\sum_{t=t}^{n}\left[f_{i} f_{t} \frac{\left(p_{i} r-R_{i}\right)\left(p_{t} r-R_{t}\right)}{\left(f_{i} p_{i}-F_{i}\right)\left(f_{t} p_{t}-F_{t}\right)}\right] .
\end{gathered}
$$

La formule déterminant l'ordre de la courbe $C_{n}$ prend cette forme

$$
c_{n}=m_{r} M_{n} \Pi_{n}(f p-F) \underset{i=t}{\stackrel{n}{\Xi}}\left[f_{i} f_{t} \frac{\left(p_{i} r-R_{i}\right)\left(p_{t} r-R_{t}\right)}{\left(f_{i} p_{i}-F_{i}\right)\left(f_{t} p_{t}-F_{t}\right)}\right] .
$$

Cette formule est exacte pour $n$, quand elle est exacte pour $n-1$. Seulement elle est valable pour $n=3$, ainsi pour $n=4$, ou elle est valable en général.

\section{$\mathrm{XV}$. Constructions générales des courbes déduites du précédent.}

52. La formule (b) renferme celle du cas général, en Jequel les courbes fondamentales ne rencontrent pas les courbes correspondantes $(p)$.

Il faut seulement y poser $F=\theta, R=\theta$ et apercevoir que la somme $\mathbf{E}$ contient toujours

$$
\frac{n(n-1)}{2}
$$

termes. Nous pouvons distinguer ces trois cas particuliers.

1. $(R)$ est un faisceau de surfaces générales et $(F)$ sont les faisceaux de plans ou les surfaces développables. La construction de la courbe est analogue à celle de la surface. 
$2 .^{\circ}(R)$ est un faisceau de plans et $\left(F^{\prime}\right)$ sont les faisceaux de surfaces générales. Ce cas nous présente trois constructions qui vont suivre.

a) Les plans tangents d'une surface $(R)$ rencontrent les courbes $\left(p_{1}\right),\left(p_{2}\right)$, $\left(p_{3}\right)$ en des points qui déterminent respectivenent des surfaces dans les faisceaux $\left(F_{1}\right),\left(F_{2}\right),\left(F_{3}\right)$. Trois de ces surfaces ainsi correspondantes, dont chacune appartient à un faisceau différent, se rencontrent en des points d'une courbe.

b) Quand un point $c$ se trouve en une telle position que, en faisant passer par lui une d̀ une surface de chacun de quatre faisceaux $\left(F_{1}\right),\left(F_{2}\right)$, $\left(F_{3}\right),\left(F_{4}\right)$, qui rencontrent les courbes $\left(p_{1}\right),\left(p_{2}\right),\left(p_{3}\right),\left(p_{4}\right)$ en des points et quand quatre de ces points pris un à un sur les courbes $(p)$ se trouvent dans un plan tangent de la surface générale $(R)$, alors le point c engendre une courbe.

c) Considérons cinq faisceaux $(F)$ et díterminons comme précédemment le point $c$ de telle manière que cinq points correspondants, pris un à un sur. chaque courbe $(p)$, se trouvent sur un plan, le point $c$ engendre une courbe.

$3 .^{\circ}$ Quand $\left(F^{\prime}\right)$ et $(R)$ sont les faisceaux de plans, nous obtenons trois constructions analogues aux précédentes, en posant les plans tangents des surfaces développables au lieu des surfaces des faisceaux.

4..$^{\circ}$ Considérons enfin le cas très important, quand chaque terme $f p-F=1$ et de même $r p-R=1$,

$$
m_{1}=m_{2}=\cdots=m_{n}=m_{r}=1 \text {. }
$$

L'ordre de la courbe $C_{n}$ est

$$
c_{n}=\sum_{i=t}^{n}\left(f_{i} f_{t}\right)
$$

Nous voyons que cette formule ne dépend pas des quantités du faisceau $(R)$ et des courbes $(p)$ qui nous servent seulement à ítablir la correspondance entre les surfaces.

Cette correspondance s'effectue de telle manière que, en prenant une à une surface dans $n-2$ faisceaux, à ces surfaces correspond une à une surface dans les deux autres faisceaux. Nous pouvons donc dire:

Quand les surfaces des $n$ faisceaux de la première dimension se correspondent de telle façon que à $(n-2)$ surfaces prises à volonté une à une dans $(n-2)$ faisceau correspond une à une surface dans les deux autres faisceaux, alors les points, par lesquels passent on même temps toutes les $n$ surfaces ainsi correspondantes, remplissent une courbe.

En multipliant deux d̀ deux ordres des surfaces des faisceaux, l'ordre de la courbe $C_{n}$ est égal à la somme de ces produits. 


\section{Surface $S_{n}$ passe par les points fondamentaux des faisceaux $(F)$.}

53. Nous avons vu, dans nos recherches sur la construction des courbes dans cas singuliers, que ces courbes rencontrent les lignes fondamentales des faisceaux $(F)$.

Cela nous amène à l'idée que la surface $S_{n}$ jouit de la même propriété, c'est-à-dire qu'elle passe par les points communs à toutes les surfaces d'un même faisceau $(F)$.

Considérons un point a par lequel passent toutes surfaces du faisceau $\left(F_{n}\right)$. Par ce point on peut faire passer plusieurs surfaces de chaque faisceau, qui rencontrent les courbes correspondantes $(p)$ en des points qui, étant pris un à un sur chacune de ces courbes, déterminent un certain nombre de surfaces $(R)$. Quelsque soient les points $p_{n}$, la surface $F_{n}$ passe toujours par le point $a$.

Ce point jouit par conséquent de la propriété que par lui passent toutes $n$ surfaces correspondantes, prises une à une dans chaque faisceau, ou, en d'autres termes, le point a appartient à la surface $S_{n}$.

54. Examinons la multiplicité d'un tel point fondamental $a$ d'un faisceau $\left(F_{k}\right)$. Afin que nous obtenions une formule la plus générale, supposons que les faisceaux $(R)$ et $\left(F_{n}\right)$ ont des points fondamentaux sur les courbes correspondantes $(p)$. Ce point a détermine

$$
m_{1}, \quad m_{2}, \ldots \quad m_{k-1}, \quad m_{k+1}, \ldots m_{n}
$$

surfaces des faisceaux

$$
\left(F_{1}\right), \quad\left(F_{2}\right), \ldots \quad\left(F_{k-1}\right), \quad\left(F_{k+1}\right), \ldots \quad\left(F_{n}\right)
$$

qui rencontrent respectivement les courbes correspondantes

$$
\left(p_{1}\right), \quad\left(p_{2}\right), \ldots \quad\left(p_{k-1}\right), \quad\left(p_{k+1}\right), \ldots \quad\left(p_{n}\right)
$$

outre les points fondamentaux en

$$
\begin{array}{cc}
f_{1} p_{1}-F_{1}, \quad f_{2} p_{2}-F_{2}, \ldots & f_{k-1} p_{k-1}-F_{k-1}, \\
f_{k+1} p_{k+1}-F_{k+1}, \ldots & f_{n} p_{n}-F_{n}
\end{array}
$$

points. En prenant un à un de ces points sur chacune de ces courbes, nous obtenons

$$
\frac{M_{n}}{m n_{k}} \cdot \frac{\Pi_{n}(f p-F)}{f_{k} p_{k}-F_{k}}
$$

groupes de tels points correspondants que chacun de ces groupes détermine $m_{r}$ 
surfaces $R$ et chacune de ces surfaces rencontre $\left(p_{k}\right)$ en $p_{k} r-R_{k}$ points, dont chacun détermine $m_{k}$ surfaces $F_{k}$ passant par $a$. De là suit que $a$ est un point multiple d'ordre

sur la surface $S_{n}$.

$$
m_{r} M_{n} \frac{\Pi_{n}(f p-F)}{f_{k} p_{k}-F_{k}}\left(p_{k} r-R_{k}\right)
$$

\section{La courbe $C_{n}$ a des points multiples} sur les courbes fondamentales des faisceanx $\left(I^{\top}\right)$.

55. Supposons que la courbe fondamentale du faisceau $\left(F_{k}\right)$ soit d'ordre $b_{k}$.

En négligeant pour un moment le faisceau $\left(F_{k}\right)$ et la courbe correspondante $\left(p_{k}\right)$, nous pouvons dériver du faisceau $(R)$ qui est de la $(n-2)^{\text {ièm" }}$ dimension à l'aide des autres $(n-1)$ faisceaux une surface $S_{n-1}$,

Cette surface rencontre la courbe fondamentale de $\left(F_{k}\right)$ en $b_{k} \cdot s_{n-1}$ points. Bien entendu, dans la construction de la surface $S_{n-1}$ avait ćté négligé le faisceau $\left(F_{k}\right)$ et non le faisceau $\left(F_{n}\right)$, ce qui a une influence à l'ordre de cette surface.

Chacun desdits points résulte d'une certaine surfacc $R$ qui rencontre la courbe $\left(p_{n}\right)$ en $r p_{k}-R_{k}$ points dont chacun détermine $m_{k}$ surfaces $\Gamma_{n}$.

Chaque tel point d'intersection est par conséquent un point multiple d'ordre

$$
\left(r p_{k}-R_{k}\right) m_{k} \text {. }
$$

Le nombre de ces points est égal à $b_{k} s_{n-1}$.

En substituant la valeur de $s_{n-1}$, nous obtenons ce théorème:

Quand le faisceau $\left(F_{k}\right)$ a une courbe fondamentale d'ordve $b_{k}$, la courbe $C_{n}$ possède sur cette courbe

$$
b_{k} m_{r} \frac{M_{n}}{m_{k}} \frac{\Pi_{n 2}(f p-F)}{f_{k} p_{k}-F_{k}}\left(\sum_{i=1}^{n} f_{i} \frac{p_{i} r-R_{i}}{f_{i} p_{i}-F_{i}}-f_{k} \frac{p_{k} r-I_{k}}{f_{k} p_{k}-F_{k}}\right)
$$

points qui sont multiples d'ordre

$$
m_{k}\left(p_{k} r-R_{k}\right)
$$

\section{Un faisceau ( $F$ ) de surfaces d'ordre $f$ possède une courbe fondamentale d'ordre $f^{2}$.}

56. On sait que ce théorème peut être demontre par une autre voie, seulement pour montrer l'importance de nos recherches nous allons en faire usage dans ce problème. 
Considérons une surface quelconque $F_{n}^{\prime}$ du faisceau $\left(F_{n}\right)$ et la surface $S_{n}$.

La surface $F^{\prime}$ rencontre $(p)$ en $f_{n} p_{n}$ points dont chacun détermine dans le faisceau $(R)$ un autre faisceau de la dimension $(n-2)$. De chacun de ces faisceaux on peut déduire à l'aide des autres $(n-1)$ faisceaux $\left(F_{1}\right),\left(F_{2}\right), \ldots$ $\left(F_{n-1}\right)$ une surface $S_{n-1}$ qui rencontre la surface $F_{n}^{\prime}$ en une courbe d'ordre $f_{n} s_{n-1}$ ou

$$
f_{n}(n-1) r m_{r} \cdot F_{n-1} M_{n-1} P_{n-1} .
$$

Puisque le nombre de ces courbes est $f_{n} p_{n}$ et la surface $S_{n}$ ne rencontre pas la surface $F_{n}^{\prime}$ en d'autres points, parce que il n'y a plus des surfaces $F_{n}$ qui coupent la surface $F_{n}^{\prime}$, la courbe d'intersection totale serait d'ordre

$$
(n-1) f_{n} r m_{r} F_{n} M_{n-1} P_{n},
$$

pendant qu'elle doit être d'ordre

$$
n r f_{n} m_{n}, F_{n} M_{n-1} P_{n} .
$$

Cela nous montre que le faisceau $\left(F_{n}\right)$ a une courbe fondamentale qui, étant la courbe d'intersection de $F^{\prime \prime}{ }_{n}$ avec $S_{n}$, est d'ordre

$$
r f_{n} m_{r} F_{n} M_{n-1} P_{n} \text {. }
$$

Ce qui précède nous apprend que, s'il y a une telle courbe, elle doit être multiple d'ordre

$$
\frac{r m_{r} F_{n} M_{n_{-1}} P_{n}}{f_{n}}
$$

et par conséquent elle est d'ordre $f_{r}^{2}$.

C. Q. F. D.

\section{Faisceaux de surfaces.}

57. Un faisceau $(R)$ de la $(n-1)^{\text {ime }}$ dimension et $n$ faisceaux $(F)$ de surfaces nous servent à la construction d'une surface $S_{n}$.

Seulement nous pouvons réciproquement d'une courbe $C$ à l'aide des $n$ faisceaux ( $F$ ) construire un faisceau $(R)$ de surfaces.

Supposons que les surfaces $R$ soient donnies par $N$ tolles conditions que par $n$ points arbitraires passent $m_{r}$ surfaces du faisceau $(R)$.

Considérons une combe $C$, les faisceaux $\left(F_{1}\right),\left(F_{2}\right), \ldots\left(F_{n}\right)$ de surfaces et leurs courbes correspondentes $\left(p_{1}\right),\left(p_{2}\right), \ldots\left(p_{n}\right)$. Un point arbitraire $c$ de la courbe $C$ détermine dans chacun des faisceaux $(F)$ un certain nombre de surfaces qui rencontrent respectivement les courbes correspondantes $\left(p_{1}\right),\left(p_{2}\right), \ldots$ 
$\left(p_{n}\right)$ en des points. En prenant un à un de ces points sur chacune de ces courbes, ces $n$ points déterminent avec les $N$ conditions données $m_{r}$ surfaces $R$. Quand le point $c$ parcourt la courbe $C$, la surface $R$ engendre un faisceau $(R)$.

Déterminons la dimension et l'indice de ce faisceau.

Pour cet effet, considérons un point arbitraire a qui détermine avec les $N$ conditions données un faisceau de la dimension $(n-1)$, du quel on peut. dériver une surface $S_{n}$ d'ordre $s_{n}$ à l'aide des faisceaux $(F)$. Cette surface rencontre la courbe $C$ en des points qui fournissent évidemment les surfaces $R$ passant par $a$.

Done

Le faisceau $(R)$ de surfaces, dérivé d'une courbe $C$ à l'aide des faisceaux $(F)$ et des courves $(p)$ jouit de la propriété que par un point arbitraire a passent $c s_{n}$ de ses surfaces ou que ce faisceau est de la première dimension et d'indice $e s_{n}$.

Il est clair que $s_{n}$ peut être une des quantités des articles précédents.

58. Quand le point $c$ parcourt une surface $S$, nous pouvons demander: quelle est la nature du faisceau de surfaces $R$ ?

Considérons le faisceau $(R)$ des articles précédents et deux points arbitraires $a, b$ qui déterminent dans $(R)$ un autre faisceau de la dimension $(n-2)$, duquel nous pouvons déduire à l'aide des faisceaux $(F)$ et des courbes $(p)$ une courbe $C_{n}$ d'ordre connu $c_{n}$.

Cette courbe perce la surface $S$ en $c_{n} s$ points qui fournissent évidemment les surfaces $R$ passant par les points $a, b$.

\section{Ainsi}

Le faisceau $(R)$ de surfaces $R$, dérivé d'une surface $S$ à l'aide des faisceaux $(F)$ et des courbes $(p)$, jouit de telle propriéte que par deux points arbitraires $a, b$ passent $c_{n} s$ surfaces $R$, ou, en d'autres termes, ce faisceau est de la dewxième dimension et d'indice $c_{n} s$.

59. Supposons que $n=3$ et que les surfaces $R$ deviennent des plans.

On sait que les faisceaux de plans de la première dimension sont les surfaces développables et les faisceaux de plans de la deuxième dimension sont les surfaces générales. Les indices de ces faisceaux sont les classes de ces surfaces.

Nous voyons donc que les théorèmes précédents renferment ces deux autres théorèmes:

1. ${ }^{\circ}$ Un point c détermine respectivement $m_{1}, m_{2}, m_{3}$ surfaces dans les faisceaux $(F)$ qui sont de la première dimension. Chacune de ces surfaces rencontre les courbes correspondantes $\left(p_{1}\right),\left(p_{2}\right),\left(p_{3}\right)$ en des points. En 
prenant un à un de ces points sur chacune de ces courbes, ces points déterminent un plan $R$.

Quand le point c parcourt une courbe $C$ d'ordre $c$, le plan $R$ enveloppe une surface développable de la classe $c s_{n}$.

Il est clair que les faisceaux $(F)$ peuvent être les surfaces développables, enveloppes des plans $F$.

2. Un point arbitraire $s$ détermine respectivement $m_{1}, m_{2}, m_{3}$ surfaces dans les faisceaux $\left(F_{1}\right),\left(F_{2}\right),\left(F_{3}\right)$ de la première dimension. Chacune de ces surfaces rencontre les courbes correspondantes respectivement en des points $p_{1}, p_{1}^{\prime}, \ldots ; p_{2}, p_{2}^{\prime}, \ldots ; p_{3}, p_{3}^{\prime}, \ldots$

En prenant un à un de ces points sur chacune de ces courbes $(p)$, ces trois points déterminent un plan $R$. Quand le point $s$ parcourt une surface $S$ d'ordre $s$, le plan $R$ enveloppe une surface générale de la classe $s c_{n}$.

Le mot générale ne signifie pas une surface générale de cette classe, seulement que cette surface n'est pas développable. Nous pourons de même dans ce théorème remplacer les faisceaux $(F)$ par des surfaces différentes.

60. Nous obtenons les faisceaux de surfaces d'une autre manière, savoir la surface $S_{n}$ remplit un faisceau.

Supposons qu'une surface $R$ est donnée par $N$ conditions et que à la détermination complete de cette surface manquent encore $n$ points.

Considérons un point arbitraire $a$ comme le point fondamental du faisceau $(R)$. De ce faisceau, qui est maintenant de la $(n-1)^{\text {irme }}$ dimension, nous pouvons déduire une surface $S_{n}$.

Quel est le lieu que remplit la surface $S_{n}$, quand le point $a$ est assujetti à parcourir une courbe $A$ d'ordre $a$ ?

Nous montrerons aisément que la surface $S_{n}$ remplit un faisceau de la première dimension, ou, en d'autres termes, que par un point arbitraire $b$ passe un certain nombre de surfaces $S_{n}$.

Considérons un tel point $b$ qui détermine $m_{1}, m_{2}, \ldots m_{n}$ surfaces $F_{1}, F_{2}, \ldots$ $F_{n}$ des faisceaux $\left(F_{1}\right),\left(F_{2}\right), \ldots\left(F_{n}\right)$.

Chacune de ces surfaces rencontre la courbe correspondante $(p)$ en

$$
f_{1} p_{1}-F_{1}, \quad f_{2} p_{2}-F_{2}, \ldots \quad f_{n} p_{n}-F_{n}
$$

points. En prenant un à un de ces points sur chacune des courbes $(p)$, ces $n$ points déterminent $m_{r}$ surfaces $R$. Nous obtenons par conséquent, en général,

surfaces $R$ d'ordre $r$.

$$
m_{r} M_{n} \Pi_{n}(f p-F)
$$


Chacune de ces surfaces rencontre la courbe $A$ en ar points, dont chacun, étant pris pour un point fondamental du faisceau, détermine une surface $S_{n}$, passant par le point $b$.

Par le point $b$ passent donc

$$
\operatorname{ar} m_{r} M_{n} \Pi_{n}(f p-F)
$$

surfaces $S_{n}$, ou, en d'autres termes, la surface $S_{n}$ remplit un faisceau de la première dimension et $d^{\prime}$ indice

$$
\operatorname{arm}, M_{n} \Pi_{n}(f p-F) \text {. }
$$

Ces surfaces sont, comme on sait, d'ordre

$$
m_{r} M_{n} \Pi_{n}(f p-F) \sum_{i}^{n}\left(f_{i} \frac{p_{i} r-R_{i}}{f_{i} p_{i}-F_{i}}\right) .
$$

\section{Faisceaux de courbes.}

61. Supposons de nouveau que le faisceau $(R)$ de surfaces $R$ soit donné par $N$ conditions de telle façon que par $n$ points arbitraires passe un certain nombre de ces surfaces.

Prenons deux points arbitraires $a, b$. Ces points déterminent un faisceau de la $(n-2)^{\mathrm{jème}}$ dimension duquel nous pouvons dériver une courbe $C_{n}$ dont l'ordre est connu.

Quel est le lieu de la courbe $C_{n}$, quand le point $b$ parcourt une courbe $B$ d'ordre $b$ ?

Il est facile de montrer que ce lieu est une surface, ou, en d'autres termes, qu' une droite arbitraire $Q$ rencontre un certain nombre de courbes $C_{n}$.

Omettons les points $a, b$. Nous pourons dériver de la droite $Q$ un faisceau de surfaces $R$, qui est de la première dimension et d'indice $c s_{n}$ ou, dans ce cas, $s_{n}$. Par le point $a$ passent par conséquent $c s_{n}$ surfaces $R$. Chacune d'elles rencontre $B$ en $b r$ points. En prenant chacun de ces points pour point fondamental du faisceau $R$, nous obtenons une courbe $S_{n}$ qui rencontre la droite $Q$.

Supposons que $R^{\prime}$ soit une des surfaces $R$, dérivée d'un point $q^{\prime}$ de la droite $Q$. La surface $R^{\prime}$ rencontre évidemment la courbe $B$ en $b r$ points, dont chacun offre une courbe qui doit passer par $q^{\prime}$. De là suit que nous obtenons de tous les points $B R^{\prime}$ d'un surface $R^{\prime}$, un seul point d'intersection sur la droite $Q$ ou que le nombre de ces points est égal au nombre de surfaces $R$ passant par a. La surface $S_{\imath}$ est donc d'ordre $s_{n}$, ce qui est vrai, parce que ce faisceau de surfaces $R$ est, proprement dit, un faisceau de la $(n-1)^{\text {ìme }}$ dimension et fournit, par conséquent, une surface d'ordre $s_{u}$. 
62. Examinons maintenant le lieu de la courbe $S_{n}$, quand le point a parcourt une courbe $A$ d'ordre $a$.

Nous trouverons que ce lieu est un faisceau de courbes, dont un certain nombre passe par un point arbitraire $c$. Le point $c$ détermine $m_{1}, m_{2}, . . m_{n}$ surfaces $\left(F_{1}\right),\left(F_{2}\right), \ldots\left(F_{n}\right)$ rencontrant respectivement les courbes correspondantes $\left(p_{1}\right),\left(p_{2}\right), \ldots\left(p_{n}\right)$ en

$$
f_{1} p_{1}-F_{1}, \quad f_{2} p_{2}-F_{2}, \ldots \quad f_{n} p_{n}-F_{n}
$$

points. En prenant un à un de ces points sur chacune des courbes $(p)$, nous obtenons

$$
M_{n} \Pi_{n}(f p-F)
$$

groupes, dont chacun détermine $m_{r}$ surfaces $R$. Chacune d'elles rencontre $A$ en $a r$ points et $B$ en $b r$ points. Considérons un de ces points, situé sur $A$ et un sur $B$; ces deux points fournissent un faisceau de la dimension $(n-2)$, qui détermine la courbe $C_{n}$ passant par le point $c$.

Chaque surface $R$ offre $a r b r$ couples de points sur les courbes $A, B$ et chacun de ces groupes fournit une courbe. Par conséquent les courbes $C_{n}$, passant par $c$, sont en nombre

$$
a b r^{2} m_{r} M_{n} \Pi_{n}(f p-F) .
$$

Nous pourons donc dire que

Etant données les surfaces $R$ par $N$ telles conditions que $n$ autres points arbitraires déterminent $m_{r}$ surfaces $R$, deux points quelconque $a, b$ déterminent un faisceau de la dimension $n-2$, duquel peut être dérivée une courbe $S_{n}$ d'ordre

$$
m_{r} \cdot M_{n} \Pi_{n}(f p-F) \sum_{i \triangleq t}^{n}\left[f_{i} f_{t} \frac{\left(p_{i} r-R_{i}\right)\left(p_{t} r-R_{t}\right)}{\left(f_{i} p_{i}-F_{i}\right)\left(f_{t} p_{t}-F_{t}\right)}\right]
$$

Quand les points $a, b$ parcourent respectivement deux courbes d'ordres $a, b$, la courbe $S_{n}$ remplit un tel faisceau que par un point arbitraire c passe

courbes $S_{n}$.

$$
a b r^{2} m_{r} M_{n} \Pi_{n}(f p-l>)
$$

\section{Sur la surface $S_{n}$} engendrée par l'intersection de deux faisceaux de surfaces.

63. En examinant l'ordre de la surface $S_{n}$, nous avons employé la surface $S_{n-1}$. Dans cet article nous allons montrer que la surface $S_{n}$ peut être obtenue comme le lieu de courbes d'intersection des surfaces $S_{n-1}$ avec les surfaces correspondantes $F_{n}$. 
Prenons un point arbitraire $p_{n}^{\prime}$ sur la courbe $\left(p_{n}\right)$. Ce point détermine dans le faisceau $(R)$ un nouveau faisceau de la $(n-2)^{\text {ieme }}$ dimension, duquel nous pouvons dériver à l'aide des autres $(n-1)$ faisceaux $(F)$ une surface $S_{n-1}^{\prime}$ qui est donc d'ordre

$$
m_{r} M_{n-1} \Pi_{n-1}(f p-F) \sum_{i}^{n-1}\left(f_{i} \frac{p_{i} r-R_{i}}{f_{i} p_{i}-F_{i}^{i}}\right)
$$

Le point $p_{n}^{\prime}$ détermine $m_{n}$ surfaces $F_{n}^{\prime}$ dans le faisceau $\left(F_{n}\right)$. Chacune de ces surfaces rencontre $S_{n-1}^{\prime}$ en une courbe qui appartient à la surface $S_{n}$.

Quand le point $p_{n}^{\prime}$ change de position sur $\left(p_{n}\right)$, nous obtenons une autre surface $S^{\prime \prime}{ }_{n-1}$ et de même autres surfaces ${F^{\prime}}_{n}$ et par conséquent aussi une autre courbe de la surface $S_{n}$.

Quand le point $p_{n}^{\prime}$ parcourt la courbe $\left(p_{n}\right)$, la surface $S_{n-1}^{\prime}$ remplit un faisceau $\left(S_{n-1}\right)$ et la surface $F_{n}^{\prime}$ remplit le faisceau $\left(F_{n}\right)$. De là suit que nous obtenons les courbes de la surface $S_{n}$ comme les intersection des faisceaux correspondants $\left(S_{n-1}\right),\left(F_{n}\right)$.

Le faisceau $\left(S_{n-1}\right)$ est selon l'article 62 de la première dimension. En substituant ar par $p_{n} r-R_{n}$ dans la formule de cet article, nous obtenons l'indice

de ce faisceau.

$$
m_{r} M_{n-1}\left(p_{n} r-R_{n}\right) \Pi_{n-1}(f p-F)
$$

64. Quand nous examinons l'ordre de la surface $S_{n}$ dans ce genre, nous trouvons que c'est le procédé que nous avons déjà employé.

A une surface $S_{n-1}$ correspondent donc $m_{n}$ surfaces $F_{n}$ et à une surface $F_{n}$ correspondent $f_{n} p_{n}-F_{n}$ surfaces $S_{n-1}^{\prime}$.

Quand les deux faisceaux de ces surfaces doivent être projectifs, c'est-àdire, quand à une surface d'un de ces faisceaux doit correspondre une seule surface de l'autre faisceau, il faut que

$$
m_{n}=1, \quad f_{n} p_{n}-F_{n}=1 ;
$$

et de plus, quand ces faisceaux doivent être de la première dimension, il faut que

$$
m_{r} M_{n-1}\left(p_{n} r-R_{n}\right) \Pi_{n-1}(f p-F)=1
$$

ou que

$$
m=1, \quad M_{n-1}=1, \quad p_{u} r-R_{n}=1
$$

et que chaque terme

$$
f p-F=1 .
$$


Nous obtenons donc que la surface $S_{n-1}^{\prime \prime}$ est d'ordre

$$
\sum_{i}^{n-1} f_{i}\left(p_{i} r-R_{i}\right)
$$

et que la surface $S_{n}$ est d'ordre

$$
\underset{i}{n-1}\left[f_{i}\left(p_{i} r-R_{i}\right)\right]+f_{n} \text {. }
$$

\section{Génération analogue d'une courbe.}

65. La même chose a lieu quant aux courbes. Prenons un point arbitraire $p_{n}^{\prime}$ sur la courbe $\left(p_{n}\right)$, qui détermine dans le faisceau $(R)$ un autre faisceau de la $(n-3)^{\text {jime }}$ dimension. Ce nouveau faisceau détermine avec les faisceaux $(F)$, excepté lo faisceau $\left(F_{n}\right)$, une courbe $C_{n-1}$.

Seulement par le point $p_{n}^{\prime}$ passent aussi plusieurs surfaces $F_{n}$ qui rencontrent la courbe $C_{n-1}$ en des points de la courbe $C_{n}$. Quand le point $p_{n}^{\prime}$ change de position sur $\left(\hat{p}_{n}\right)$, la courbe $C_{n-1}$ et les surfaces $F_{n}$ le font aussi. Quand le point $p_{n}^{\prime}$ parcourt la courbe $\left(p_{n}\right)$, la courbe $C_{n+1}$ engendre, suivant le précédent, une surface $S_{n-1}$ et les surfaces $F_{n}$ remplissent le faisceau $\left(F_{n}\right)$.

Nous obtenons ainsi les points de la courbe $C_{n}$ comme les points d'intersection des courbes $C_{n-1}$ avec les surfaces correspondantes $F_{n}$, la correspondance étant de telle sorte, que à une courbe $C_{n-1}$ correspond un certain nombre de surfaces $F_{n}$, et réciproquement à une surface $F_{n}$ correspond une certaine courbe $C_{n-1}$.

Il est intéressant que tous les points de la courbe $C_{n}$ se trouvent sur la surface $S_{n-1}$.

En omettant un autre faisceau, par exemple $\left(F_{k}\right)$ de surfaces $F_{k}$, et en construisant du faisceau $R$ une surface $S_{n k}$, la courbe $C_{n}$ doit se trouver toute entière sur cette surface. Cette courbe est donc l'intersection desdites surfaces $S_{n-1}, S_{n k}$.

Nous obtenons ainsi la courbe $C_{n}$ comme la ligne d'intersection de plusieurs surfaces. En négligeant un faisceau $(F)$ et sa courbe correspondante $(p)$, nous obtenons toujours une surface de $(R)$. Il y en a $n$ et toutes ces surfaces passent par la même courbe $C_{n}$ qui fait une partie de leur courbe d'intersection. 